\title{
The Flow Separation of Peristaltic Transport for Maxwell Fluid between Two Coaxial Tubes
}

\author{
S. Z. A. Husseny, ${ }^{1,2}$ Y. Abd elmaboud, ${ }^{3,4}$ and Kh. S. Mekheimer ${ }^{2}$ \\ ${ }^{1}$ Mathematical Department, Faculty of Science, King Abdulaziz University, Jeddah 21589, Saudi Arabia \\ ${ }^{2}$ Mathematics Department, Faculty of Science, Al-Azhar University, Nasr City, Cairo 11884, Egypt \\ ${ }^{3}$ Mathematics Department, Faculty of Science and Arts, King Abdulaziz University (KAU), Khulais 21921, Saudi Arabia \\ ${ }^{4}$ Mathematics Department, Faculty of Science, Al-Azhar University (Assiut Branch), Assiut 71524, Egypt \\ Correspondence should be addressed to Y. Abd elmaboud; yass_math@yahoo.com
}

Received 10 January 2014; Revised 27 April 2014; Accepted 6 May 2014; Published 28 May 2014

Academic Editor: Victor Kovtunenko

Copyright (c) 2014 S. Z. A. Husseny et al. This is an open access article distributed under the Creative Commons Attribution License, which permits unrestricted use, distribution, and reproduction in any medium, provided the original work is properly cited.

\begin{abstract}
We study the peristaltic mechanism of an incompressible non-Newtonian biofluid (namely, Maxwell model) in the annular region between two coaxial tubes. The inner tube represents the endoscope tube. The system of the governing nonlinear PDE is solved by using the perturbation method to the first order in dimensionless wavenumber. The modified Newton-Raphson method is used to predict the flow separation points along the peristaltic wall and the endoscope tube. The results show that the presence of the endoscope (catheter) tube in the artery increases the pressure gradient and shear stress. Such a result seems too reasonable from the physical and medical point of view.
\end{abstract}

\section{Introduction}

Peristalsis is produced by sequential waves of contractions in an elastic channel (tube), which push their fluid forward. In the urinary system, peristaltic movement is due to spontaneous muscular contractions of the ureteral wall which drives urine from the kidneys to the bladder through the ureters. Peristalsis is an important property of many biological systems which transports biofluids by its propulsive movements. It is responsible for the movement of chyme in the gastrointestinal tract, intrauterine fluid motion, vasomotion of the small blood vessels and in many glandular ducts. A number of analytical studies of peristaltic flows of different fluids have been reported [1-5].

Non-Newtonian fluids have wide-range applications, so they receive a great attention from scientists. The flows of non-Newtonian fluids are important because of their technological significance. Moreover, physiological flows indicate that non-Newtonian viscoelastic rheology is the correct way of properly describing the peristaltic flow through channels and tubes. Among many models, which have been employed to describe the non-Newtonian behavior exhibited by certain real fluids, Oldroyd fluid has obtained a special status; it includes elastic and memory effects exhibited by dilute solutions. Oldroyd fluid has been extensively used in many applications, and also results of simulations fit experimental data quite well $[6,7]$.

Recently, peristaltic motion of non-Newtonian fluids has been an important subject in the field of chemical, biomedical, and environmental engineering and science. A considerable amount of the literature has been reported [8-17].

Endoscope and catheters are very important tools for medical diagnosis and they have many clinical applications. The endoscope now is a very important tool used for determining the real causes responsible for many problems in the human organs through which the fluids are transported by peristaltic pumping such as, stomach and small intestine. Also from the fluid dynamic point of view, there is no difference between an endoscope and catheter. In medicine, a catheter is a tube that can be inserted into a body cavity, duct, or vessel. Catheters thereby allow drainage or injection of fluids. The process of inserting a catheter is called catheterization. Also, the insertion of an endoscope (catheter) 
in an artery will alter the flow field and modify the pressure distribution. There are several investigations that studied the effect of an endoscope on peristaltic transport for different fluids [18-22].

So far, no attempt has been made to understand the effect of endoscope on the peristaltic motion for the Maxwell model which is a subclass of Oldroyd model. Therefore the main purpose of the present paper is to study the mathematical modeling of peristaltic transport of flow in a gap between two coaxial tubes, filled with an incompressible non-Newtonian fluid (Maxwell model). The inner tube is rigid (endoscope) and the outer one has wave trains moving independently. In this paper, we are concerned with viscoelastic flows governed by the Maxwell model. The mathematical modeling of this problem is explained below in brief.

\section{Basic Equations}

The basic equations governing the motion of Oldroyd fluid take the form

$$
\begin{gathered}
\nabla \cdot \mathbf{V}^{\prime}=0, \\
\rho \frac{D \mathbf{V}^{\prime}}{D t}=\nabla \cdot \mathbf{T}^{\prime},
\end{gathered}
$$

where $\rho$ is the density, $\mathbf{V}^{\prime}$ is the velocity vector, $D / D t$ denotes the material derivative, and $\mathbf{T}^{\prime}$ is the Cauchy stress tensor. The Cauchy stress $\mathbf{T}^{\prime}$ for an incompressible Oldroyd fluid can be expressed as

$$
\begin{gathered}
\mathbf{T}^{\prime}=-p^{\prime} \mathbf{I}+\mathbf{S}^{\prime}, \\
\mathbf{S}^{\prime}+\Gamma\left(\frac{d \mathbf{S}^{\prime}}{d t}-\mathbf{L}^{\prime} \mathbf{S}^{\prime}-\mathbf{S}^{\prime} \mathbf{L}^{\prime T}\right) \\
=\mu\left[\mathbf{A}_{1}^{\prime}+\nu\left(\frac{d \mathbf{A}_{1}^{\prime}}{d t}-\mathbf{L}^{\prime} \mathbf{A}_{1}^{\prime}-\mathbf{A}_{1}^{\prime} \mathbf{L}^{\prime T}\right)\right],
\end{gathered}
$$

where $p^{\prime}$ is the pressure, $\mu$ is the coefficient of viscosity, $\mathbf{S}^{\prime}$ is the extra stress, $\Gamma$ is the relaxation time, and $\nu$ is the retardation time. The Rivlin-Ericksen tensors are defined as

$$
\mathbf{A}_{1}^{\prime}=\left(\nabla \mathbf{V}^{\prime}\right)+\left(\nabla \mathbf{V}^{\prime}\right)^{T}, \quad \mathbf{L}^{\prime}=\nabla \mathbf{V}^{\prime}
$$

It is worth mentioning that this model includes the Maxwell model for $v=0$ and the classical linear case for $\Gamma=v=0$.

\section{Formulation of the Problem and Mathematical Model}

In this paper, the flow of the Maxwell fluid is considered through the gap between coaxial tubes. The inner tube is rigid and the outer has a sinusoidal wave traveling down its walls. The geometry of the walls surface (Figure 1) is

$$
\begin{gathered}
R_{1}^{\prime}=a_{1}, \\
R_{2}^{\prime}=a_{2}+b \sin \left(\frac{2 \pi}{\lambda}\left(Z^{\prime}-c t^{\prime}\right)\right),
\end{gathered}
$$

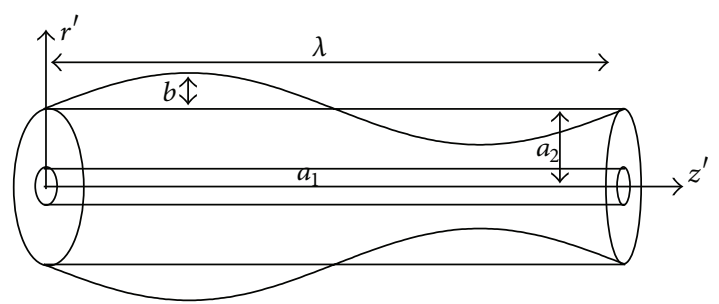

FIGURE 1: Geometry of the problem.

where $a_{1}$ is the radius of the inner tube, $a_{2}$ is the radius of the outer tube at any axial distance $Z^{\prime}$, and $b$ is the amplitude of the wave. Introducing a wave frame $\left(r^{\prime}, z^{\prime}\right)$ moving with velocity $c$ away from the fixed frame $\left(R^{\prime}, Z^{\prime}\right)$ by the transformation

$$
\begin{array}{ll}
z^{\prime}=Z^{\prime}-c t^{\prime}, & r^{\prime}=R^{\prime}, \\
w^{\prime}=W^{\prime}-c, & u^{\prime}=U^{\prime},
\end{array}
$$

where $\left(u^{\prime}, w^{\prime}\right)$ and $\left(U^{\prime}, W^{\prime}\right)$ are the velocity components in the wave and fixed frame, respectively. After using this transformation then the mathematical formulation of this problem results in the following differential equations:

$$
\begin{aligned}
\frac{\partial u^{\prime}}{\partial r^{\prime}}+\frac{\partial w^{\prime}}{\partial z^{\prime}}+\frac{u^{\prime}}{r^{\prime}}=0, & \\
\rho\left[u^{\prime} \frac{\partial u^{\prime}}{\partial r^{\prime}}+w^{\prime} \frac{\partial u^{\prime}}{\partial z^{\prime}}\right]= & -\frac{\partial p^{\prime}}{\partial r^{\prime}} \\
& -\left[\frac{1}{r^{\prime}} \frac{\partial}{\partial r^{\prime}}\left(r^{\prime} S_{11}^{\prime}\right)-\frac{S_{22}^{\prime}}{r^{\prime}}+\frac{\partial S_{13}^{\prime}}{\partial z^{\prime}}\right], \\
\rho\left[u^{\prime} \frac{\partial w^{\prime}}{\partial r^{\prime}}+w^{\prime} \frac{\partial w^{\prime}}{\partial z^{\prime}}\right]= & -\frac{\partial p^{\prime}}{\partial z^{\prime}}-\left[\frac{1}{r^{\prime}} \frac{\partial}{\partial r^{\prime}}\left(r^{\prime} S_{13}^{\prime}\right)+\frac{\partial S_{33}^{\prime}}{\partial z^{\prime}}\right] .
\end{aligned}
$$

The constitutive equations of Maxwell fluid are

$$
\begin{gathered}
S_{11}^{\prime}+\Gamma\left[u^{\prime} \frac{\partial S_{11}^{\prime}}{\partial r^{\prime}}+w^{\prime} \frac{\partial S_{11}^{\prime}}{\partial z^{\prime}}-2 S_{11}^{\prime} \frac{\partial u^{\prime}}{\partial r^{\prime}}-2 S_{13}^{\prime} \frac{\partial u^{\prime}}{\partial z^{\prime}}\right]=-\mu \dot{\gamma}_{11}^{\prime}, \\
S_{13}^{\prime}+\Gamma\left[u^{\prime} \frac{\partial S_{13}^{\prime}}{\partial r^{\prime}}+w^{\prime} \frac{\partial S_{13}^{\prime}}{\partial z^{\prime}}-S_{33}^{\prime} \frac{\partial u^{\prime}}{\partial z^{\prime}}-S_{11}^{\prime} \frac{\partial w^{\prime}}{\partial r^{\prime}}+\frac{u^{\prime}}{r^{\prime}} S_{13}^{\prime}\right] \\
=-\mu \dot{\gamma}^{\prime}{ }_{13}, \\
S_{22}^{\prime}+\Gamma\left[u^{\prime} \frac{\partial S_{22}^{\prime}}{\partial r^{\prime}}+w^{\prime} \frac{\partial S_{22}^{\prime}}{\partial z^{\prime}}-\frac{2 u^{\prime}}{r} S_{22}^{\prime}\right]=-\mu \dot{\gamma}_{22}^{\prime}, \\
S_{33}^{\prime}+\Gamma\left[u^{\prime} \frac{\partial S_{33}^{\prime}}{\partial r^{\prime}}+w^{\prime} \frac{\partial S_{33}^{\prime}}{\partial z^{\prime}}-2 S_{33}^{\prime} \frac{\partial w^{\prime}}{\partial z^{\prime}}-2 S_{13}^{\prime} \frac{\partial w^{\prime}}{\partial r^{\prime}}\right] \\
=-\mu \dot{\gamma}^{\prime}{ }_{33},
\end{gathered}
$$

where $\rho$ is the density, $P^{\prime}$ is the pressure, $S_{i j}^{\prime}$ are the components of the extra stress tensor and $\dot{\gamma}^{\prime}{ }_{i j}$ are the components 
of strain-rate tensor, and $\Gamma$ is the relaxation time. The system of nonlinear differential equations has to be solved with the following boundary conditions:

$$
\begin{gathered}
w^{\prime}=-c, \quad u^{\prime}=0, \quad \text { at } r^{\prime}=r_{1}^{\prime}, \\
w^{\prime}=-c, \quad u^{\prime}=-c \frac{d r_{2}^{\prime}}{d z^{\prime}}, \quad \text { at } r^{\prime}=r_{2}^{\prime} .
\end{gathered}
$$

Consider the following nondimensional variables and parameters:

$$
\begin{gathered}
r=\frac{r^{\prime}}{a_{2}}, \quad z=\frac{z^{\prime}}{\lambda}, \quad w=\frac{w^{\prime}}{c}, \\
u=\frac{\lambda u^{\prime}}{a_{2} c}, \quad p=\frac{a_{2}^{2}}{\lambda \mu c} p^{\prime}, \quad r_{1}=\frac{r_{1}^{\prime}}{a_{2}}=\epsilon, \quad \phi=\frac{b}{a_{2}}, \\
S_{i j}=\frac{a_{2} S_{i j}^{\prime}}{c \mu}, \quad r_{2}=\frac{r_{2}^{\prime}}{a_{2}}=1+\phi \sin (2 \pi z), \\
\operatorname{Re}=\frac{\rho c a_{2}}{\mu}, \quad \delta=\frac{a_{2}}{\lambda}, \quad \epsilon=\frac{a_{1}}{a_{2}}, \quad \mathrm{Wi}=\frac{c \Gamma}{a_{2}},
\end{gathered}
$$

where $\phi$ is the amplitude ratio, Re is the Reynolds number, $\delta$ is the dimensionless wave number and $\mathrm{Wi}$ is the Weissenberg number, and $\epsilon$ is the radius ratio (the ratio between the radius of the inner tube and the radius of the outer). To proceed, we nondimensionalize ((4), (6)-(7)); this yields

$$
\begin{gathered}
r_{1}=\epsilon, \\
r_{2}=1+\phi \sin (2 \pi z), \\
\frac{1}{r} \frac{\partial}{\partial r}(r u)+\frac{\partial w}{\partial z}=0, \\
\operatorname{Re} \delta^{3}\left[u \frac{\partial u}{\partial r}+w \frac{\partial u}{\partial z}\right] \\
\operatorname{Re} \delta\left[u \frac{\partial w}{\partial r}+w \frac{\partial w}{\partial z}\right]=-\frac{\partial p}{\partial z}\left[\frac{1}{r} \frac{\partial}{\partial r}\left(r S_{11}\right)-\frac{S_{22}}{r}+\delta \frac{\partial S_{13}}{\partial z}\right], \\
S_{11}+\operatorname{Wi} \delta\left[u \frac{\partial S_{11}}{\partial r}+w \frac{\partial S_{11}}{\partial z}-2 S_{11} \frac{\partial u}{\partial r}-2 \delta S_{13} \frac{\partial u}{\partial z}\right] \\
=-2 \delta \frac{\partial u}{\partial r},
\end{gathered}
$$

$$
\begin{gathered}
S_{13}+\mathrm{Wi}\left[\delta\left(u \frac{\partial S_{13}}{\partial r}+w \frac{\partial S_{13}}{\partial z}-\delta S_{33} \frac{\partial u}{\partial z}+\frac{u}{r} S_{13}\right)-S_{11} \frac{\partial w}{\partial r}\right] \\
=-\left(\delta^{2} \frac{\partial u}{\partial z}+\frac{\partial w}{\partial r}\right) \\
S_{22}+\mathrm{Wi} \delta\left[u \frac{\partial S_{22}}{\partial r}+w \frac{\partial S_{22}}{\partial z}-\frac{2 u}{r} S_{22}\right]=-2 \delta \frac{u}{r} \\
S_{33}+\mathrm{Wi}\left[\delta\left(u \frac{\partial S_{33}}{\partial r}+w \frac{\partial S_{33}}{\partial z}-2 S_{33} \frac{\partial w}{\partial z}\right)-2 S_{13} \frac{\partial w}{\partial r}\right] \\
=-2 \delta\left(\frac{\partial w}{\partial z}\right) .
\end{gathered}
$$

The non-dimensional boundary conditions will be

$$
\begin{gathered}
w=-1, \quad u=0, \quad \text { at } r=\epsilon, \\
w=-1, \quad u=-\frac{d r_{2}}{d z}, \quad \text { at } r=r_{2} .
\end{gathered}
$$

\section{Rate of Volume Flow in the Annulus}

The instantaneous volume flow rate in the fixed frame is given by

$$
Q=2 \pi \int_{R_{1}^{\prime}}^{R_{2}^{\prime}} R^{\prime} W^{\prime} d R^{\prime}
$$

where $R_{2}^{\prime}$ is a function of $Z^{\prime}, t^{\prime}$. The rate of volume flow in the wave frame is given by

$$
q=2 \pi \int_{r_{1}^{\prime}}^{r_{2}^{\prime}} r^{\prime} w^{\prime} d r^{\prime}
$$

where $r_{2}^{\prime}$ is a function of $z^{\prime}$ only. If we substitute (5) into (20) and make use of (21), we find that the two rates of volume flow are related through

$$
Q=q+\pi c\left(r_{2}^{\prime 2}-r_{1}^{\prime 2}\right)
$$

The time mean flow over a period $T$ at a fixed position $Z^{\prime}$ is defined as

$$
Q^{\prime}=\frac{1}{T} \int_{0}^{T} Q d t
$$

Substituting (22) into (23) and integrating, we get

$$
Q^{\prime}=q+\pi c a_{2}^{2}\left(1+\frac{\phi^{2}}{2}\right)-\pi c r_{1}^{\prime 2} .
$$

On defining the dimensionless time-mean flows $\bar{Q}$ and $F$, respectively, in the fixed and wave frame as

$$
\bar{Q}=\frac{Q^{\prime}}{\pi c a_{2}^{2}}, \quad F=\frac{q}{\pi c a_{2}^{2}},
$$


one finds that (24) may be written as

$$
\bar{Q}=F+1+\frac{\phi^{2}}{2}-\epsilon^{2}
$$

where

$$
F=2 \int_{r_{1}}^{r_{2}} r w d r
$$

\section{Perturbation Solution}

It is evident that $((12)-(18))$ are highly nonlinear, and, as such, it is not possible to obtain the solutions in a closed form. In order to solve the present problem, we expanded the flow quantities in a power series of the small parameter $\delta$ as follows:

$$
\begin{gathered}
u=u_{0}+\delta u_{1}+\delta^{2} u_{2}+\cdots, \\
w=w_{0}+\delta w_{1}+\delta^{2} w_{2}+\cdots, \\
p=p_{0}+\delta p_{1}+\delta^{2} p_{2}+\cdots, \\
\tau_{i j}=S_{i j}^{(0)}+\delta S_{i j}^{(1)}+\delta^{2} S_{i j}^{(2)}+\cdots \quad \text { where } i, j=1,2,3, \\
F=F_{0}+\delta F_{1}+\delta^{2} F_{2}+\cdots .
\end{gathered}
$$

If we substitute (28) into ((12)-(19)) and separate the terms of different order in $\delta$, we obtain the following systems of partial differential equations together with boundary conditions as follows.

System of Order Zero. Consider

$$
\begin{aligned}
& 0=-\frac{\partial p_{0}}{\partial r} \\
& 0=-\frac{\partial p_{0}}{\partial z}-\left(\frac{1}{r} \frac{\partial}{\partial r}\left(r S_{13}^{0}\right)\right), \\
& S_{11}^{0}=0, \\
& S_{13}^{0}+\mathrm{Wi}\left(-S_{11}^{0} \frac{\partial w_{0}}{\partial r}\right)=-\frac{\partial w_{0}}{\partial r}, \\
& S_{22}^{0}=0, \\
& S_{33}^{0}+\mathrm{Wi}\left(-2 S_{13}^{0} \frac{\partial w_{0}}{\partial r}\right)=0,
\end{aligned}
$$

with the boundary conditions:

$$
\begin{aligned}
& w_{0}=-1, \quad u_{0}=0, \quad \text { at } r=r_{1}=\epsilon, \\
& w_{0}=-1, \quad u_{0}=-\frac{d r_{2}}{d z}, \quad \text { at } r=r_{2} .
\end{aligned}
$$

System of Order One. Consider

$$
\begin{gathered}
0=-\frac{\partial p_{1}}{\partial r}-\frac{1}{r} \frac{\partial}{\partial r}\left(r S_{11}^{0}\right)-\frac{1}{r} S_{22}^{0}, \\
\operatorname{Re}\left[u_{0} \frac{\partial w_{0}}{\partial r}+w_{0} \frac{\partial w_{0}}{\partial z}\right]=-\frac{\partial p_{1}}{\partial z}-\left(\frac{1}{r} \frac{\partial}{\partial r}\left(r S_{13}^{1}\right)\right)+\frac{\partial S_{33}^{0}}{\partial z} \\
S_{11}^{1}+\mathrm{Wi}\left(u_{0} \frac{\partial S_{11}^{0}}{\partial r}+w_{0} \frac{\partial S_{11}^{0}}{\partial z}-2 S_{11}^{0} \frac{\partial u_{0}}{\partial r}\right)=-2 \frac{\partial u_{0}}{\partial r} \\
S_{13}^{1}+\mathrm{Wi}\left[u_{0} \frac{\partial S_{13}^{0}}{\partial r}+w_{0} \frac{\partial S_{13}^{0}}{\partial z}+\frac{u_{0}}{r} S_{13}^{0}-S_{11}^{0} \frac{\partial w_{1}}{\partial r}-S_{11}^{1} \frac{\partial w_{0}}{\partial r}\right] \\
\left.=-\frac{\partial w_{1}}{\partial r}, \quad-2 S_{13}^{0} \frac{\partial w_{1}}{\partial r}-2 S_{13}^{1} \frac{\partial w_{0}}{\partial r}\right]=-2 \frac{\partial w_{0}}{\partial z} \\
S_{22}^{1}+\mathrm{Wi}\left[u_{0} \frac{\partial S_{22}^{0}}{\partial r}+w_{0} \frac{\partial S_{22}^{0}}{\partial z}-\frac{2 u_{0}}{r} S_{22}^{0}\right]=-2 \frac{u_{0}}{r} \\
S_{33}^{1}+\mathrm{Wi}\left[u_{0} \frac{\partial S_{33}^{0}}{\partial r}+w_{0} \frac{\partial S_{33}^{0}}{\partial z}-2 S_{33}^{0} \frac{\partial w_{0}}{\partial z}\right.
\end{gathered}
$$

with the boundary conditions:

$$
\begin{gathered}
w_{1}=0, \quad u_{1}=0, \quad \text { at } r=r_{1}=\epsilon, \\
w_{1}=0, \quad u_{1}=0, \quad \text { at } r=r_{2} .
\end{gathered}
$$

Solving the above sets of equations with the corresponding boundary conditions, we get the following.

Zero-Order Problem. Consider

$$
\begin{gathered}
w_{0}(r, z)=-1+\log [r] \Omega_{7}+\Omega_{8}+r^{2} \Omega_{9}, \\
u_{0}(r, z)=\Omega_{3}-r \Omega_{4}+r \log [r] \Omega_{5}-r \Omega_{6}, \\
\frac{d p_{0}}{d z}=\left(8\left(F_{0}-\epsilon^{2}+r_{2}^{2}\right)\left(\log [\epsilon]-\log \left[r_{2}\right]\right)\right) \\
\times\left(\left[( \epsilon ^ { 2 } - r _ { 2 } ^ { 2 } ) \left(-\epsilon^{2}+r_{2}^{2}+\epsilon^{2} \log [\epsilon]+r_{2}^{2} \log [\epsilon]\right.\right.\right. \\
\left.\left.\left.-\epsilon^{2} \log \left[r_{2}\right]-r_{2}^{2} \log \left[r_{2}\right]\right)\right]\right)^{-1} .
\end{gathered}
$$

We point out that the zero order solution coincides with the solution obtained by Mekheimer and Abd elmaboud [22] and is still independent of the viscoelastic effects.

First-Order Problem. Substituting the zeroth-order solution ((33) and (34)) into the equation of motion obtained for first 
order, one finds that the solution of the first-order problem will be in the form

$$
\begin{aligned}
w_{1}(r, z)= & \frac{d p_{1}}{d z}\left(\frac{r^{2}}{4}+\frac{\left(\epsilon^{2} / 4-r_{2}^{2} / 4\right) \log [r]}{\log \left[r_{2}\right]-\log [\epsilon]}\right. \\
& \left.\quad+\frac{\epsilon^{2} \log \left[r_{2}\right]-r_{2}^{2} \log [\epsilon]}{4\left(\log [\epsilon]-\log \left[r_{2}\right]\right)}\right) \\
& +r^{2} \Omega_{17}+r^{2} \log [r] \Omega_{18} \\
& +r^{4}\left(\Omega_{19}+\log [r] \Omega_{20}\right)+\Omega_{21} \\
& +\log [r] \Omega_{22}+\frac{\Omega_{15}+2 \mathrm{Wi} \log [r] \Omega_{5} \Omega_{7}}{r} \\
& +\frac{2}{9} r^{3} \operatorname{Re} \Omega_{3} \Omega_{9}+r\left(\Omega_{16}+4 \mathrm{Wi} \log [r] \Omega_{5} \Omega_{9}\right) \\
& +\frac{1}{4} r^{2} \operatorname{Re} \log [r]^{2} \Omega_{7} \Omega_{7}^{\prime}+\frac{1}{2} \mathrm{Wi} \log [r]^{2} \Omega_{7} \Omega_{7}^{\prime} \\
& +\frac{1}{36} r^{6} \operatorname{Re} \Omega_{9} \Omega_{9}^{\prime},
\end{aligned}
$$

$$
u_{1}(r, z)=c_{6}
$$

$$
\begin{aligned}
& +\frac{1}{r}\left\{-\frac{1}{36} r^{6}\left(6 \Omega_{19}^{\prime}-\Omega_{20}\right)\right. \\
& -r\left(\Omega_{15}^{\prime}-2 \mathrm{Wi}\left(\Omega_{7} \Omega_{5}^{\prime}+\Omega_{5} \Omega_{7}^{\prime}\right)\right) \\
& -\frac{2}{45} r^{5} \operatorname{Re}\left(\Omega_{9} \Omega_{3}^{\prime}+\Omega_{3} \Omega_{9}^{\prime}\right) \\
& -\frac{1}{9} r^{3}\left(3 \Omega_{16}^{\prime}-4 \mathrm{Wi}\left(\Omega_{9} \Omega_{5}^{\prime}+\Omega_{5} \Omega_{9}^{\prime}\right)\right) \\
& -\frac{1}{16} r^{2}\left(r^{2} \operatorname{Re}+4 \mathrm{Wi}\right)(\log [r])^{2} \\
& \times\left(\Omega_{7}^{\prime 2}+\Omega_{7} \Omega_{7}^{\prime \prime}\right) \\
& -\frac{1}{128} r^{4}\left(32 \Omega_{17}^{\prime}-8 \Omega_{18}^{\prime}\right. \\
& \left.+\operatorname{Re}\left(\Omega_{7}^{\prime 2}+\Omega_{7} \Omega_{7}^{\prime \prime}\right)\right) \\
& -\frac{r^{2}}{\left(16 r_{2}\left(\log [\epsilon]-\log \left[r_{2}\right]\right)^{2}\right)} \\
& \times\left(\frac{d p_{1}}{d z} r_{2}^{\prime}(1+2 \log [\epsilon])\right. \\
& \times\left(\epsilon^{2}+r_{2}^{2}\left(2 \log \left[r_{2}\right]-1-2 \log [\epsilon]\right)\right) \\
& +2 r_{2}\left(\log [\epsilon]-\log \left[r_{2}\right]\right)^{2} \\
& \left.\times\left(4 \Omega_{21}^{\prime}-2 \Omega_{22}^{\prime}+\mathrm{Wi}\left(\Omega_{7}^{\prime 2}+\Omega_{7} \Omega_{7}^{\prime \prime}\right)\right)\right)
\end{aligned}
$$$$
-\log [r]\left[\frac{1}{6} r^{6} \Omega_{20}^{\prime}\right.
$$$$
+2 r \mathrm{Wi}\left(\Omega_{7} \Omega_{5}^{\prime}+\Omega_{5} \Omega_{7}^{\prime}\right)
$$$$
+\frac{4}{3} r^{3} \mathrm{Wi}\left(\Omega_{9} \Omega_{5}^{\prime}+\Omega_{5} \Omega_{9}^{\prime}\right)
$$$$
+\frac{1}{32} r^{4}\left(8 \Omega_{18}^{\prime}-\operatorname{Re}\left(\Omega_{7}^{\prime 2}+\Omega_{7} \Omega_{7}^{\prime \prime}\right)\right)
$$$$
+\frac{r^{2}}{\left(8 r_{2}\left(\log [\epsilon]-\log \left[r_{2}\right]\right)^{2}\right)}
$$$$
\times\left(\frac { d p _ { 1 } } { d z } r _ { 2 } ^ { \prime } \left(-\epsilon^{2}\right.\right.
$$$$
+r_{2}^{2}(1+2 \log [\epsilon]
$$$$
\left.\left.-2 \log \left[r_{2}\right]\right)\right)
$$$$
+2 r_{2}\left(\log [\epsilon]-\log \left[r_{2}\right]\right)^{2}
$$$$
\times\left(2 \Omega_{22}^{\prime}\right.
$$

$$
\left.\left.\left.-\mathrm{Wi}\left(\Omega_{7}^{\prime 2}+\Omega_{7} \Omega_{7}^{\prime \prime}\right)\right)\right)\right]
$$

$$
\left.-\frac{r^{8} \operatorname{Re}}{288}\left(\Omega_{9}^{\prime 2}+\Omega_{9} \Omega_{9}^{\prime \prime}\right)\right\}
$$

$$
\begin{aligned}
\frac{d p_{1}}{d z}=1( & -\frac{\epsilon^{4}}{8}+\frac{\epsilon^{4}}{8\left(\log [\epsilon]-\log \left[r_{2}\right]\right)} \\
& -\frac{\epsilon^{2} r_{2}^{2}}{4\left(\log [\epsilon]-\log \left[r_{2}\right]\right)} \\
& \left.+\frac{r_{2}^{4}\left(1+\log [\epsilon]-\log \left[r_{2}\right]\right)}{8\left(\log [\epsilon]-\log \left[r_{2}\right]\right)}\right)^{-1} \\
\times\{ & -F_{1}-2 \epsilon \Omega_{15}-\frac{2}{3} \epsilon^{3} \Omega_{16}-\frac{1}{2} \epsilon^{4} \Omega_{17} \\
& +\frac{1}{8} \epsilon^{4} \Omega_{18}-\frac{1}{2} \epsilon^{4} \log [\epsilon] \Omega_{18}-\frac{1}{3} \epsilon^{6} \Omega_{19} \\
& +\frac{1}{18} \epsilon^{6} \Omega_{20}-\frac{1}{3} \epsilon^{6} \log [\epsilon] \Omega_{20} \\
& +\frac{1}{18} r_{2}^{6}\left(6 \Omega_{19}+\left(-1+6 \log \left[r_{2}\right]\right) \Omega_{20}\right) \\
& -\epsilon^{2} \Omega_{21}+\frac{1}{2} \epsilon^{2} \Omega_{22}-\epsilon^{2} \log [\epsilon] \Omega_{22} \\
& +4 \mathrm{Wi} \epsilon \Omega_{5} \Omega_{7}-4 \mathrm{Wi} \epsilon \log [\epsilon] \Omega_{5} \Omega_{7} \\
& +2 r_{2}\left(\Omega_{15}+2 \mathrm{Wi}\left(-1+\log \left[r_{2}\right]\right) \Omega_{5} \Omega_{7}\right) \\
& \frac{4}{45} \epsilon^{5} \Omega_{3} \Omega_{9}+\frac{4}{45} \operatorname{Re} r_{2}^{5} \Omega_{3} \Omega_{9}
\end{aligned}
$$




$$
\begin{aligned}
& +\frac{8}{9} \mathrm{Wi} \epsilon^{3} \Omega_{5} \Omega_{9}-\frac{8}{3} \mathrm{Wi} \epsilon^{3} \log [\epsilon] \Omega_{5} \Omega_{9} \\
& +\frac{2}{9} r_{2}^{3}\left(3 \Omega_{16}+4 \mathrm{Wi}\left(-1+3 \log \left[r_{2}\right]\right) \Omega_{5} \Omega_{9}\right) \\
& -\frac{1}{4} \mathrm{Wi} \epsilon^{2} \Omega_{7} \Omega_{7}^{\prime}-\frac{1}{64} \operatorname{Re} \epsilon^{4} \Omega_{7} \Omega_{7}^{\prime} \\
& +\frac{1}{2} \mathrm{Wi} \epsilon^{2} \log [\epsilon] \Omega_{7} \Omega_{7}^{\prime} \\
& +\frac{1}{16} \operatorname{Re} \epsilon^{4} \log [\epsilon] \Omega_{7} \Omega_{7}^{\prime} \\
& -\frac{1}{2} \mathrm{Wi} \epsilon^{2} \log [\epsilon]^{2} \Omega_{7} \Omega_{7}^{\prime} \\
& -\frac{1}{8} \operatorname{Re} \epsilon^{4} \log [\epsilon]^{2} \Omega_{7} \Omega_{7}^{\prime} \\
& +\frac{1}{4} r_{2}^{2}\left(4 \Omega_{21}+\left(-2+4 \log \left[r_{2}\right]\right) \Omega_{22}\right. \\
& +\mathrm{Wi}\left(1+2\left(-1+\log \left[r_{2}\right]\right)\right. \\
& \left.\left.\times \log \left[r_{2}\right]\right) \Omega_{7} \Omega_{7}^{\prime}\right) \\
& +\frac{1}{64} r_{2}^{4}\left(32 \Omega_{17}+8\left(-1+4 \log \left[r_{2}\right]\right) \Omega_{18}\right. \\
& +\operatorname{Re}\left(1-4 \log \left[r_{2}\right]\right. \\
& \left.\left.+8 \log \left[r_{2}\right]^{2}\right) \Omega_{7} \Omega_{7}^{\prime}\right) \\
& \left.-\frac{1}{144} \operatorname{Re} \epsilon^{8} \Omega_{9} \Omega_{9}^{\prime}+\frac{1}{144} \operatorname{Re} r_{2}^{8} \Omega_{9} \Omega_{9}^{\prime}\right\} \text {, }
\end{aligned}
$$

where $\left(^{\prime}\right)$ represents differentiation with respect to $z$, and the coefficients $\Omega_{i j}$ and $c_{6}$ that appear in the whole paper are listed in the appendix. The total expressions for the velocities and the pressure gradient up to first order can be obtained by substituting $((33)-(38))$ and $F_{0}=F-\delta F_{1}$ into (28) and neglecting the terms greater than $O(\delta)$.

The pressure rise $\Delta p$, in nondimensional form, is given by

$$
\Delta p=\int_{0}^{\Lambda} \frac{d p}{d z} d z
$$

where $\Lambda=L / \lambda$.

The integral in (39) is not integrable in a closed form; it is evaluated numerically with aid of the MATHEMATICA programme.

\section{The Flow Separation Points}

In cases of separation from bodies of smooth geometrical form, the surface streamlines are observed to leave (separate) the surface more or less tangentially. In the case where the boundary layer is laminar, in sufficient momentum exchange takes, the flow is unable to adjust to the increasing pressure and separates from the surface. Contemplation of the velocity field shows that it is plausible to identify the point of separation $\left(z_{s}\right)$ on the walls with points where the circulation vanishes (the vorticity equal zero) [23]. A condition used to prognosticate the separation in boundary-layer theory is to set the vorticity equal to zero on the boundary walls [10], as

$$
\eta_{z}=\frac{\partial u}{\partial z}-\frac{\partial w}{\partial r}, \quad \text { at } r=\epsilon, r_{2}
$$

(the result of (40) is omitted because of large output). We can solve the resultant equation $\left(\left.\eta_{z}\right|_{r=r_{2}}=0\right.$ and $\left.\left.\eta_{z}\right|_{r=\epsilon}=0\right)$ numerically using the modified Newton-Raphson method [24] to get the flow separation points $z_{s}$. The flow separation points $z_{s}$ with different values of the amplitude ratio $\phi$ in the tube $(\epsilon=0)$ and in the annulus $(\epsilon \neq 0)$ are summarized in Table 1.

The table shows that there are two values of separation points $z_{s}$. An important point here is that there is no separation when $\phi=0$ on the tube wall and on the annulus walls. Moreover, the separation points are different in tube than those in annulus. Also, the separation points are different from the amplitude ratio to the other one and from radius ratio to the other one. The effect of various parameters of interest on the wall vorticity will be discussed in the next section.

\section{Numerical Results and Discussion}

This section is divided into three subsections. In the first subsection, the effects of various parameters on the velocity distribution and wall vorticity are investigated. The pumping characteristics and shear stresses are discussed in the second subsection. The trapping phenomenon is illustrated in the last subsection. The emerging parameters of our analysis are the Reynolds number Re, wave number $\delta$, the Weissenberg number $\mathrm{Wi}$, the radius ratio $\epsilon$, the amplitude ratio $\phi$, and the average flow rate $\bar{Q}$. These have easy physical interpretations: $\delta$ is a measure of how large the semi depth of the peristaltic motion is, as compared to its wavelength. It is an aspect ratio and thus an expression of shallowness. The Reynolds number Re is formed with the wave speed, the amplitude, and the kinematic viscosity of the Newtonian part of the constitutive behavior. Wi measures the elastic contributions of the stress behavior. $\epsilon$ gives the ratio between the radius of the inner tube and the radius of the outer tube of the annulus. $\phi$ is a measure of how large the wave amplitude of the peristaltic wave is as compared to the radius of the outer tube.

7.1. Axial Velocity, Wall Vorticity, and Flow Separation. This subsection describes the influences of various emerging parameters of our analysis on the transverse distributions of the longitudinal velocity at the gap of the annulus $w(r, z)$ and the wall vorticity $\eta_{z}$ on the outer and inner tube walls. Figures $2,3,4,5,6,7,8$, and 9 display the effects of $\operatorname{Re}, \delta, \mathrm{Wi}, \epsilon, \phi$, and $\bar{Q}$ on $w(r, z)$ and $\eta_{z}$. From Figures 2 and 3, owing to the limit of the no-slip boundary condition in (19), the velocity at wall tubes has the same value $w=-1$ in the wave frame for all values of the parameters; that is, the fluid velocity at the annulus walls moves with an opposite velocity to that of the 
TABLE 1: Computed values of the flow separation points $z_{s}$ for different values of amplitude ratio $\phi$ at $\bar{Q}=1, \operatorname{Re}=10, \mathrm{Wi}=0.01$, and $\delta=0.01$.

\begin{tabular}{cccc}
\hline \multirow{\phi}{*}{$\phi$} & Tube $\epsilon=0$ & Annulus $\epsilon=0.05$ & Annulus $\epsilon=0.1$ \\
& $z_{s}$ & $z_{s}$ & $z_{s}$ \\
\hline \multirow{2}{*}{0} & $\ldots$ & $\ldots$ & $\ldots$ \\
& $\ldots$ & $\ldots$ & $\ldots$ \\
\hline \multirow{2}{*}{0.1} & 0.6045724 & 0.6504434 & 0.6504434 \\
& 0.8975483 & 0.8702141 & 0.8615617 \\
\hline \multirow{2}{*}{0.2} & 0.5678108 & 0.5987454 & 0.6078304 \\
& 0.9194012 & 0.9139087 & 0.9015790 \\
\hline \multirow{2}{*}{0.3} & 0.5575255 & 0.5987454 & 0.6078304 \\
& 0.9102325 & 0.9076357 & 0.8959550 \\
\hline \multirow{2}{*}{0.4} & 0.5479702 & 0.6050183 & 0.6162664 \\
& 0.9014732 & 0.9043910 & 0.8901146 \\
\hline \multirow{2}{*}{0.5} & 0.5327034 & 0.5991780 & 0.6162664 \\
& 0.904884 & 0.9072031 & 0.8901146 \\
\hline
\end{tabular}

wave that propagates on the outer wall of the annulus. As we expected, the magnitudes of forward and backward flows (in the annulus gap) increase when the wave number $\delta$ (i.e, small wave length for the peristaltic wave, so the amplitude of the wave increases) and Reynolds number Re increase. Moreover, Figure 2, indicates that the location of the reflux phenomenon in the flow depends upon the magnitudes of Re and $\delta$. Effects of the Weissenberg number $\mathrm{Wi}$ and the radius ratio $\epsilon$ are illustrated in Figure 3. The curves for Wi match together and a slight difference occurs only for $\epsilon=0.01$ near the inner wall. For $\epsilon=0.1$ the data are identical for Wi values. It is worth mentioning that the shear stress decreases with an increase in the relaxation parameter $\Gamma, \mathrm{Wi}=c \Gamma / a_{2}$, so that the velocity increases. However, an increase of the radius ratio $\epsilon$ produces a decrease of the velocity and more back flow occurs.

The variation of the flow vorticity at the outer and inner tubes along the annulus is illustrated in Figures 4-9 for different values of the emerging parameters. These figures show that the vorticity increases in the wider part of the annulus, $z \in(0,0.5)$, while it decreases as going to the narrow part, $z \epsilon(0.51,1)$, and separation occurs at the outer tube but at the inner one there are no separation points appearing (Figures 7-9). Moreover, the separation points positions are in the narrow part of the annulus. In solid annulus (no peristaltic $\phi=0$,) we observe that the vorticity is constant along the annulus and, therefore, there are no separation points (this result is compatible with the results in Table 1.). By increasing the value of the amplitude ratio $\phi$ (peristaltic transport), separation points appear but this depends on the position $z$.

7.2. The Pumping Characteristics and Shear Stresses. This subsection describes the influences of various emerging parameters of our analysis on the axial pressure gradient $(d p / d z)$, the pressure rise per wavelength $(\Delta p)$, and the shear stresses at the annulus walls. The effects of these parameters are shown in Figures 10, 11, 12, 13, 14, 15, and 16. Figures 10 and 11 illustrate that in the wider part of the channel

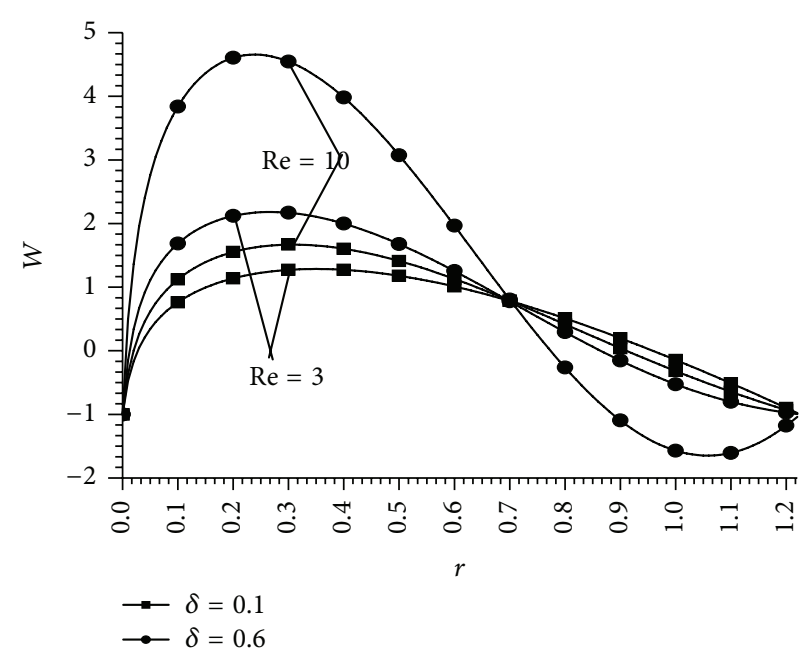

FIGURE 2: The velocity distribution $w$, across the annulus with different values of Re and $\delta$ at $\epsilon=0.01, \mathrm{Wi}=0.01, \bar{Q}=1.5, \phi=0.4$, and $z=0.1$, where $r \in\left[\epsilon, r_{2}\right]$.

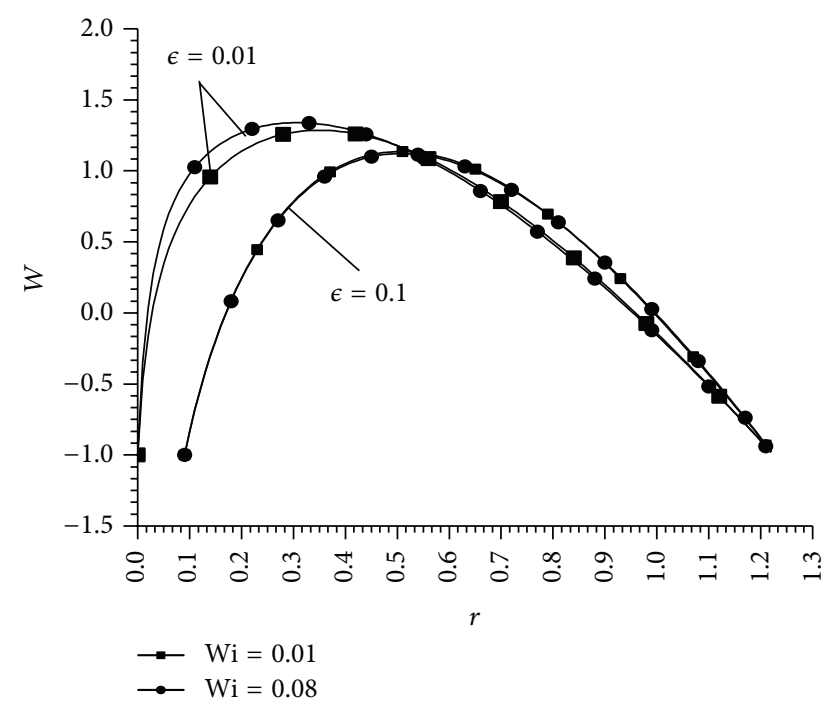

FIGURE 3: The velocity distribution $w$, across the annulus with different values of $\epsilon$ and $\mathrm{Wi}$ at $\operatorname{Re}=3, \delta=0.1, \bar{Q}=1.5, \phi=0.4$, and $z=0.1$, where $r \in\left[\epsilon, r_{2}\right]$.

$z \in[0,0.6]$ and $[0.9,1.0]$ the pressure gradient is relatively small; that is, the flow can easily pass without imposition of large pressure gradient. However, in a narrow part of the channel $z \in[0.6,0.9]$ a much larger pressure gradient is required to maintain the same flux to pass it, especially for the narrowest position near $z=0.8$. This is in good agreement with the physical situation. Also from these two figures we observe the effect of $\epsilon$ and $\delta$ on the pressure gradient, for fixed values of the other parameters, where the amplitude of $d p / d x$ increases with increasing $\epsilon$ and $\delta$. Figure 11 shows that the amplitude of the axial pressure gradient decreases as Reynolds number increases. Reynolds number is the ratio of inertial to viscous forces and with the increase of Reynolds number the viscous forces decrease so the velocity increases 


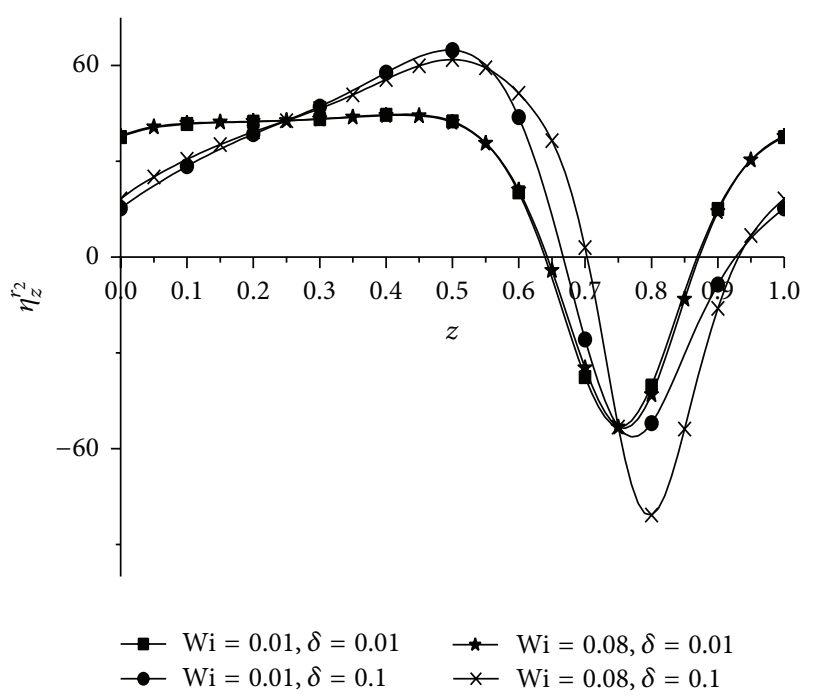

FIGURE 4: Variation of wall vorticity $\eta_{z}^{r_{2}}$ versus $z$ with different values of $\delta$ and Wi at $\operatorname{Re}=5, \epsilon=0.01, \bar{Q}=1.5$, and $\phi=0.4$.

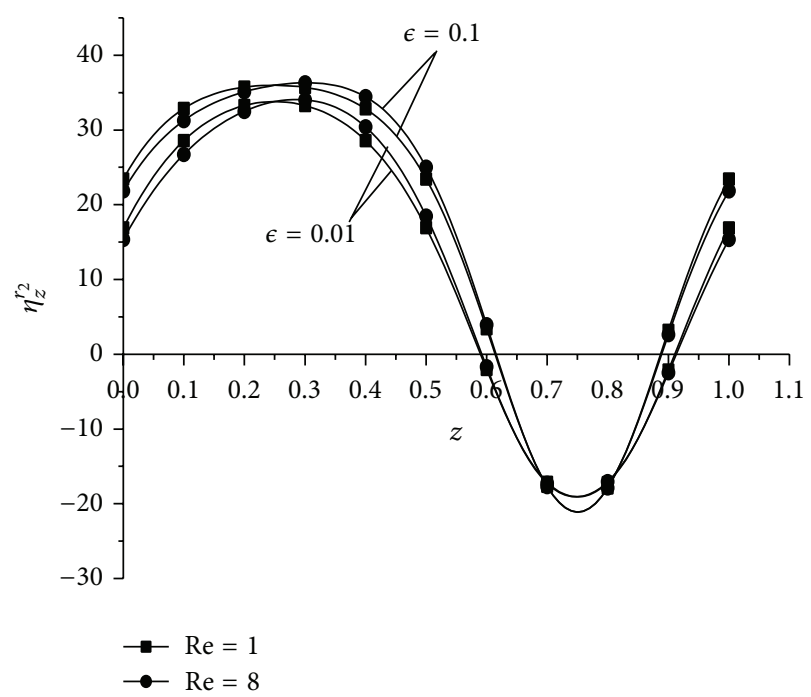

FIGURE 5: Variation of wall vorticity $\eta_{z}^{r_{2}}$ versus $z$ with different values of $\epsilon$ and $\operatorname{Re}$ at $\mathrm{Wi}=0.01, \delta=0.1, \overline{\mathrm{Q}}=1$, and $\phi=0.4$.

and so the pressure gradient decreases. Also, as the Wi increases (stresses decreases) the pressure gradient increases. In Figure 12, the pressure rise $\Delta p$ is illustrated in terms of the wave amplitude $\phi$ of the wall disturbance with various values of the radius ratio $\epsilon$. Firstly, it is obvious that with increasing occlusion $\phi$ the pressure drop increases; that is, an increase of the pressure gradient is needed to push the same flux to pass the gap annulus. Secondly, the pressure rise for an annulus is higher than that for a tube. Figure 12 is sectored so that the upper right-hand quadrant denotes the region of peristaltic pumping where $\bar{Q}>0$ (positive pumping) and $\Delta p>0$ (adverse pressure gradient). The lower righthand quadrant denotes the region of augmented pumping where $\bar{Q}>0$ (positive pumping) and $\Delta p<0$ (favorable

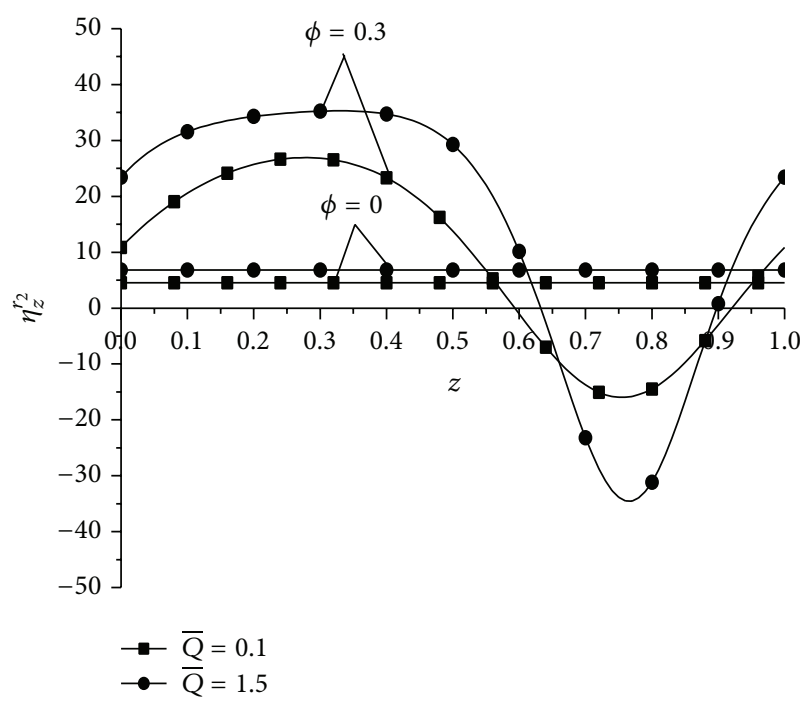

FIGURE 6: Variation of wall vorticity $\eta_{z}^{r_{2}}$ versus $z$ with different values of $\bar{Q}$ and $\phi$ at $\mathrm{Wi}=0.02, \delta=0.1, \epsilon=0.01$, and $\operatorname{Re}=1$.

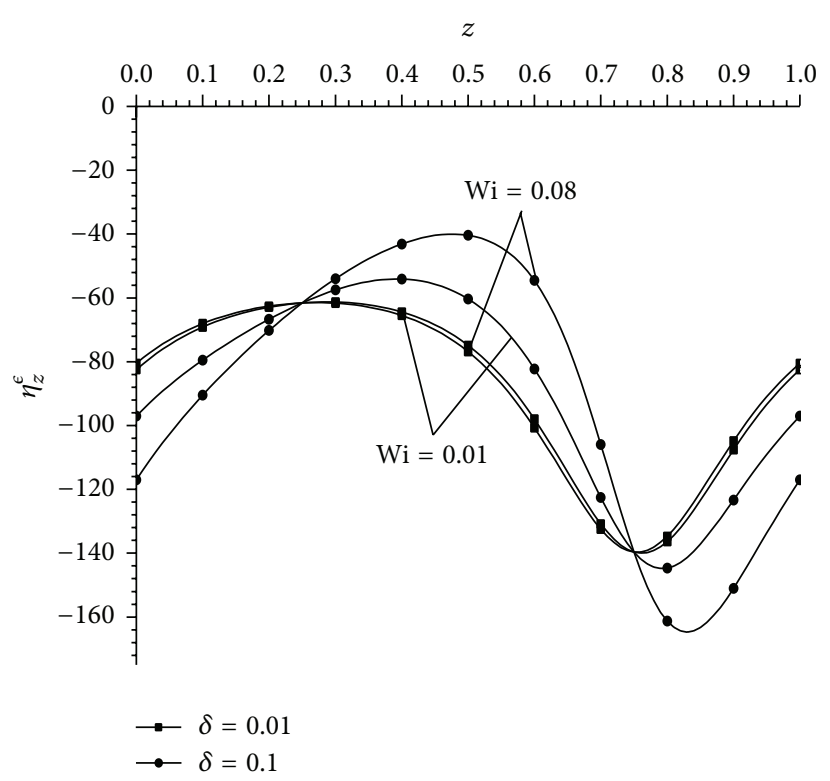

FIGURE 7: Variation of wall vorticity $\eta_{z}^{\epsilon}$ versus $z$ with different values of $\delta$ and Wi at $\operatorname{Re}=5, \epsilon=0.01, \bar{Q}=1.5$, and $\phi=0.4$.

pressure gradient) and the upper left-hand quadrant denotes the region of retrograde pumping (or backward pumping) where $\bar{Q}<0$ and $\Delta p>0$ (adverse pressure gradient); in this region the flows is opposite to the direction of the peristaltic motion. It is clear that the peristaltic pumping region becomes wider as the radius ratio $\epsilon$, amplitude ratio $\phi$ (the ratio between wave amplitude and the radius of the outer tube), increases.

The influences of various parameters of our analysis on shear stress $\tau_{13}$ at the outer and inner walls are shown in Figures 13-16. The shear stress $\tau_{13}$ in the outer tube varies along the annulus from small value in the wider part to large 


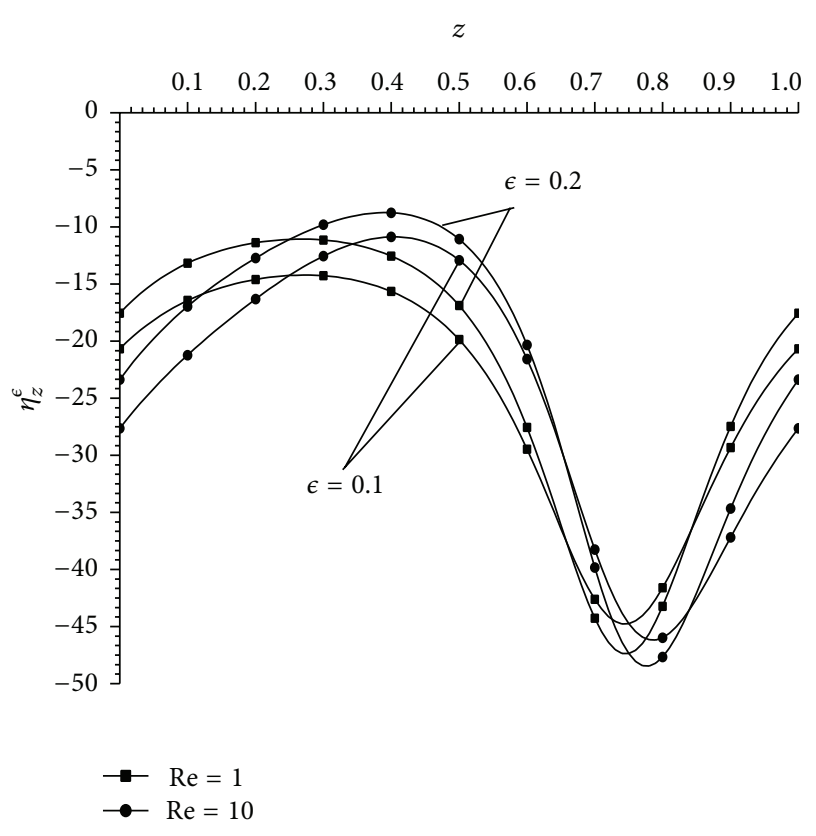

FIGURE 8: Variation of wall vorticity $\eta_{z}^{\epsilon}$ versus $z$ with different values of $\epsilon$ and $\mathrm{Re}$ at $\mathrm{Wi}=0.01, \delta=0.1, \overline{\mathrm{Q}}=1$, and $\phi=0.4$.

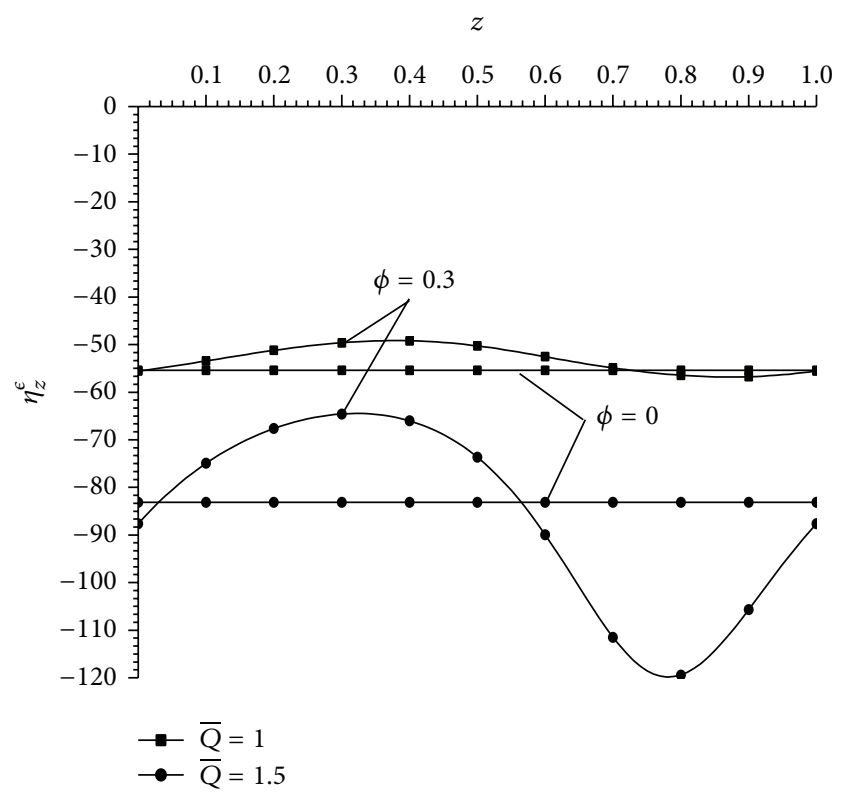

FIGURE 9: Variation of wall vorticity $\eta_{z}^{\epsilon}$ versus $z$ with different values of $\bar{Q}$ and $\phi$ at $\mathrm{Wi}=0.02, \delta=0.1, \epsilon=0.01$, and $\mathrm{Re}=1$.

value in the narrow part but the shear stress in the inner tube has an opposite behavior. The maximum amplitude of the shear stress $\tau_{13}$ increases with an increase in both $\epsilon$ and $\delta$ as displayed in Figure 13. Figure 14 shows that there is no variation in the shear stress $\tau_{13}$ along the annulus in the case of solid wall annulus $(\phi=0)$, but an apparent variation is noticed at $\phi>0$ (peristaltic movement). The figure also shows that, in the peristaltic movement case $(\phi>0)$, there is an apparent variation in the shear stress $\tau_{13}$ with various values

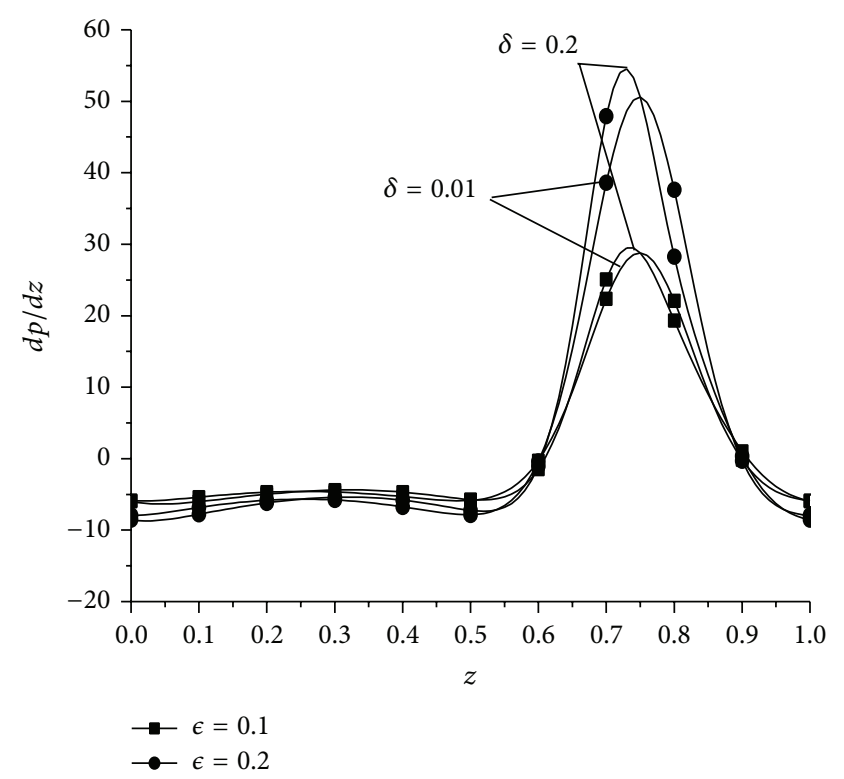

FIGURE 10: Variation of pressure gradient versus $z$ with different values of $\delta$ and $\epsilon$ at $\mathrm{Wi}=0.05, \bar{Q}=0.5, \phi=0.4$, and $\mathrm{Re}=2$.

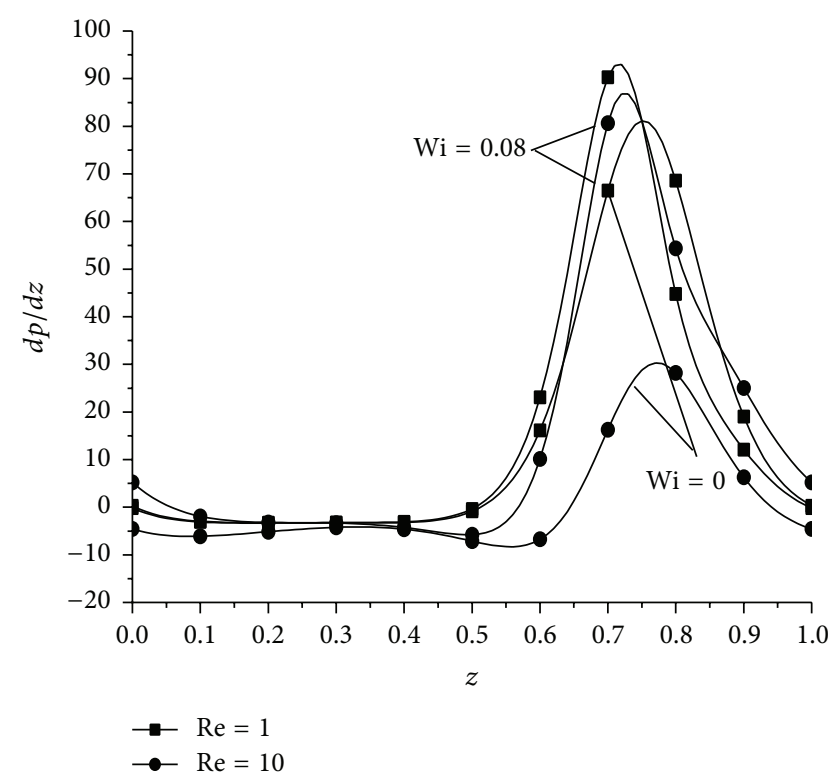

FIGURE 11: Variation of pressure gradient versus $z$ with different values of Wi and Re at $\delta=0.1, \bar{Q}=0.1, \phi=0.4$, and $\epsilon=0.1$.

of the Weissenberg number Wi along the annulus. The inverse results are seen through Figures 15 and 16 for the shear stress at the inner wall.

7.3. Trapping Phenomenon. An important phenomenon in peristaltic motion is trapping. In a wave frame, the streamline under particular conditions split to trap a bolus which moves as a whole with the speed of the peristaltic wave. The effects of the Reynolds number Re, Weissenberg parameter Wi, the wave number $\delta$, and radius ratio $\epsilon$ on trapping can be observed through Figures 17, 18, 19, and 20. The effect of 


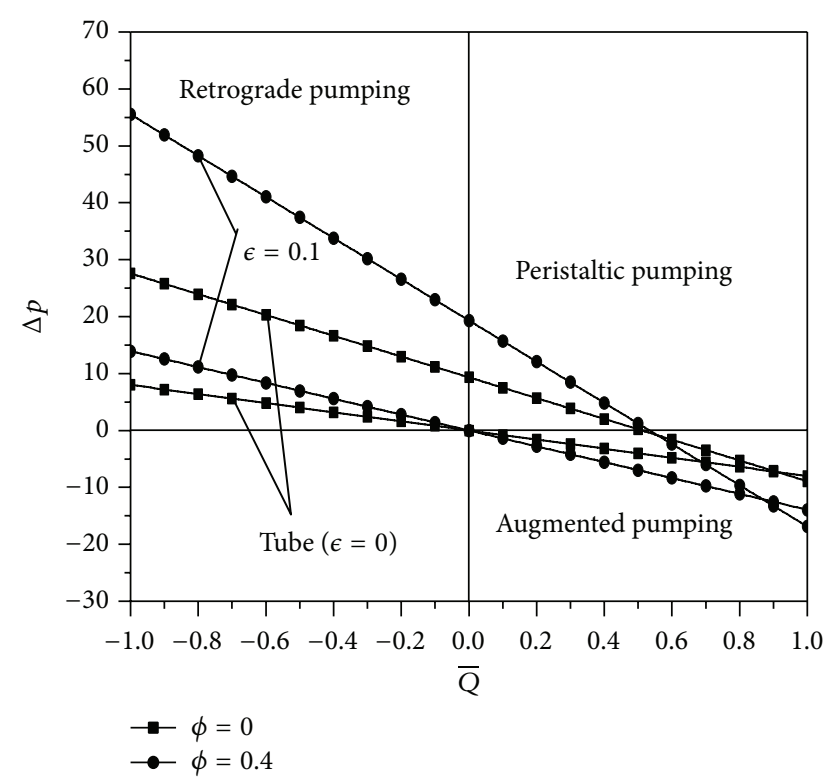

FIGURE 12: The variation of pressure rise over the length $\Delta p$ with $\bar{Q}$ for different values of $\phi$ and $\epsilon$ at $\mathrm{Wi}=0.01, \operatorname{Re}=10$, and $\delta=0.08$.

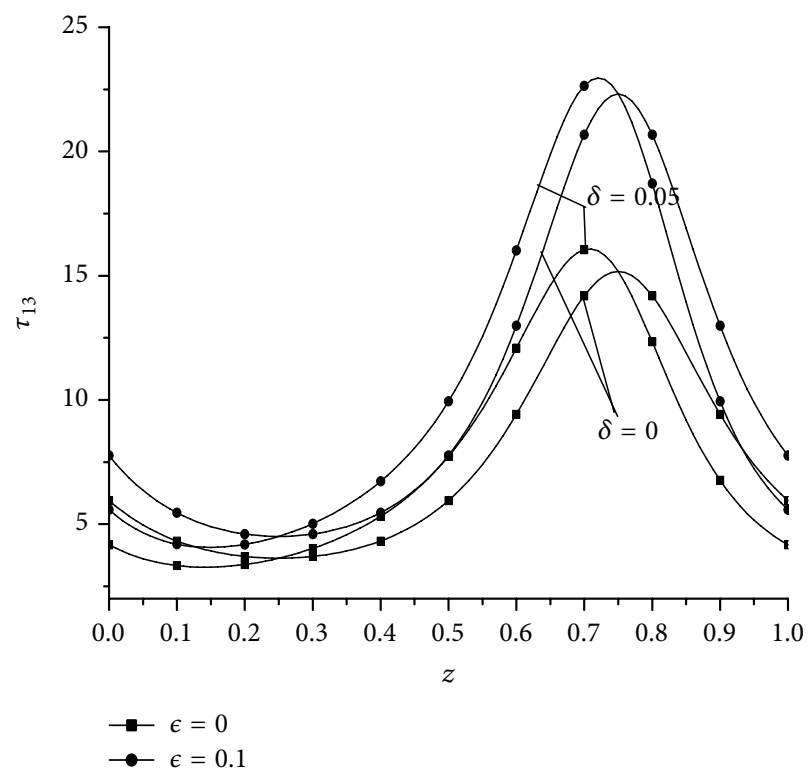

FIgURE 13: Wall shear stress $\tau_{13}$ at the outer wall versus $z$ with different values of $\delta$ and $\epsilon$ at $\mathrm{Wi}=0.01, \bar{Q}=1.5, \phi=0.4$, and $\operatorname{Re}=10$.

inertia on trapping is shown in Figure 17. The figure displays that the streamlines corresponding to $\mathrm{Re}=0$ in Figure 17(a) are in the form of trapped bolus and by elevating the values of Re more trapped boluses appear as in Figure 17(b). With further increase in $\mathrm{Re}$, we find the presence of a second eddy as seen in Figure 17(c). We can see that there is no substantial difference to be seen on trapping by changing Weissenberg parameter Wi as shown in Figure 18. In order to see the effect of the curvature $\delta$ on trapping we have plotted Figure 19. We see that the shape of the trapped bolus is deformed by

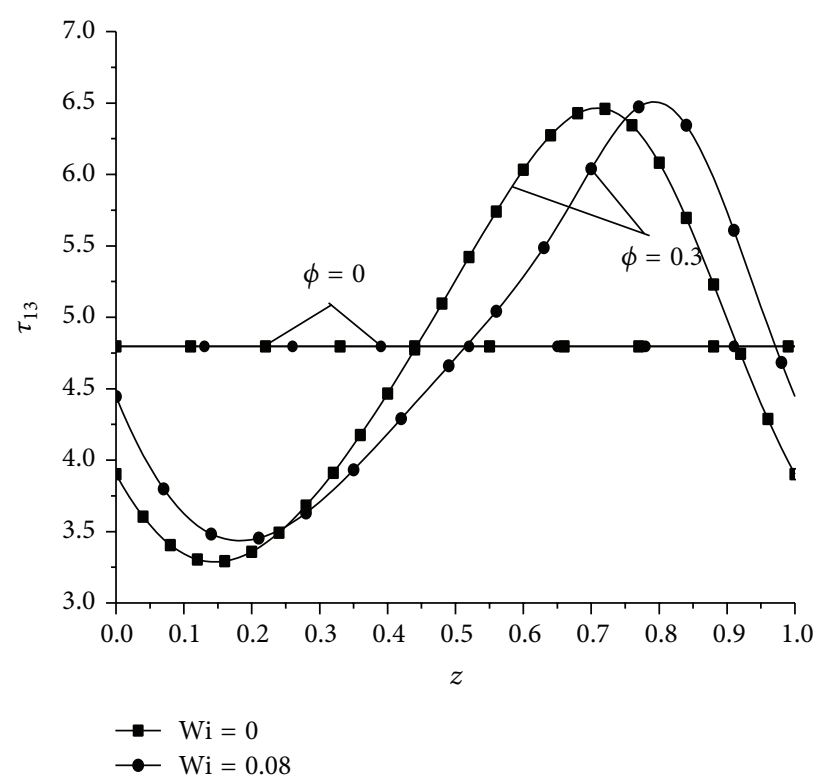

FIGURE 14: Wall shear stress $\tau_{13}$ at the outer wall versus $z$ with different values of $\phi$ and Wi at $\epsilon=0.1, \bar{Q}=1, \delta=0.08$, and $\operatorname{Re}=10$.

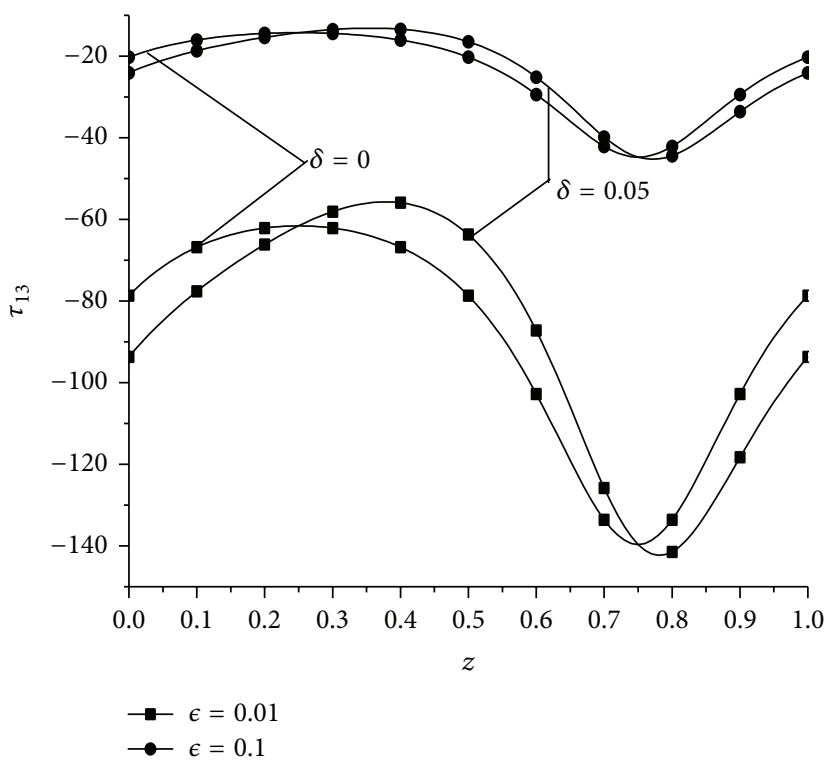

FIGURE 15: Wall shear stress $\tau_{13}$ at the inner wall versus $z$ with different values of $\delta$ and $\epsilon$ at $\mathrm{Wi}=0.01, \bar{Q}=1.5, \phi=0.4$, and $\operatorname{Re}=10$.

increasing the values of the dimensionless wave number $\delta$. The streamlines graph for different values of radius ratio $\epsilon$ is plotted in Figure 19. The "trapping" phenomena occur for $\epsilon=$ 0.2 (Figure 19(a)). But for a further decrease in $(\epsilon=0)$ (tube), the trapped bolus is seen with large size and the trapping range increases gradually as in Figure 19(c).

\section{Concluding Results}

The mathematical model of peristaltic transport of flow in a gap between two coaxial tubes filled with Maxwell fluid is 


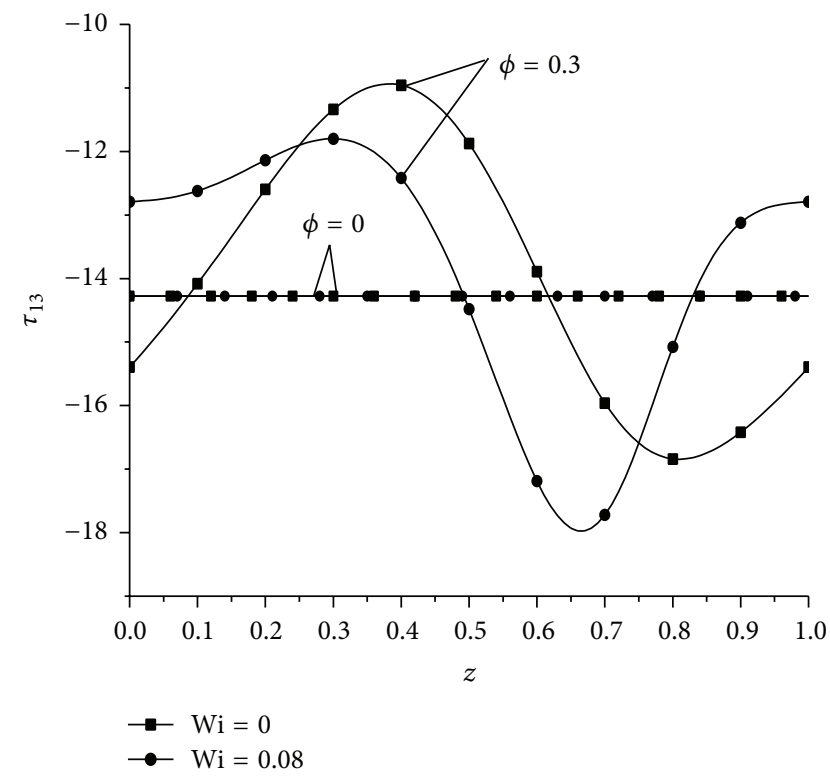

FIGURE 16: Wall shear stress $\tau_{13}$ at the inner wall versus $z$ with different values of $\phi$ and Wi at $\epsilon=0.1, \bar{Q}=1, \delta=0.08$, and $\operatorname{Re}=10$.

studied analytically. The main findings can be summarized as follows.

(i) There exist two values of a separation points at the outer wall of the annulus. An important result is that there is no separation when $\phi=0$ (solid wall) on both the tube wall and the annulus walls. Moreover, the separation points are different in the tube than those in the annulus.

(ii) In the narrow part of the annulus, the maximum amplitude of the pressure gradient for Maxwell fluid is different from the Newtonian fluid.

(iii) Inertial force effect on the peristaltic flow is stronger for the case of larger amplitude ratio.

(iv) The shear stress $\tau_{13}$ in the outer tube along the annulus varies from small values in the wider part to large values in the narrow part, but the shear stress in the inner tube behaves oppositely.

(v) The presence of the endoscope tube $(\epsilon \neq 0)$ in an artery increases the pressure gradient and shear stress. Such a result seems reasonable from the physical and medical point of view.

(vi) The presence of a second eddy is seen by increasing the values of the Reynolds number.

\section{Appendix}

$$
\begin{aligned}
& \Omega_{1}=2 r_{2}\left(\epsilon^{2}-r_{2}^{2}\right)\left(\epsilon^{2}\left(-1+\log [\epsilon]-\log \left[r_{2}\right]\right)\right. \\
& \left.+r_{2}^{2}\left(1+\log [\epsilon]-\log \left[r_{2}\right]\right)\right) \\
& \times\left(\log [\epsilon]-\log \left[r_{2}\right]\right), \\
& \Omega_{2}=2 \epsilon^{2} r_{2}^{2}+\epsilon^{4}\left(-1+\log [\epsilon]-\log \left[r_{2}\right]\right) \\
& +r_{2}^{4}\left(-1-\log [\epsilon]+\log \left[r_{2}\right]\right), \\
& \Omega_{3}=\left(\epsilon\left(F_{0}-\epsilon^{2}+r_{2}^{2}\right)\right. \\
& \left.\times\left(\epsilon^{2}+r_{2}^{2}\left(-1-2 \log [\epsilon]+2 \log \left[r_{2}\right]\right)\right) r_{2}^{\prime}\right) \\
& \times\left(\Omega_{1}\right)^{-1} \text {, } \\
& \Omega_{4}=\left(\left(F_{0}-\epsilon^{2}+r_{2}^{2}\right)\right. \\
& \left.\times\left(\epsilon^{2}+r_{2}^{2}\left(-1-2 \log [\epsilon]+2 \log \left[r_{2}\right]\right)\right) r_{2}^{\prime}\right) \\
& \times\left(\Omega_{1}\right)^{-1} \text {, } \\
& \Omega_{5}=\left(2\left(F_{0}-\epsilon^{2}+r_{2}^{2}\right)\right. \\
& \left.\times\left(\epsilon^{2}+r_{2}^{2}\left(-1-2 \log [\epsilon]+2 \log \left[r_{2}\right]\right)\right) r_{2}^{\prime}\right) \\
& \times\left(\Omega_{1}\right)^{-1} \text {, } \\
& \Omega_{6}=\left(2\left(F_{0}-\epsilon^{2}+r_{2}^{2}\right) \log [\epsilon]\right. \\
& \left.\times\left(\epsilon^{2}+r_{2}^{2}\left(-1-2 \log [\epsilon]+2 \log \left[r_{2}\right]\right)\right) r_{2}^{\prime}\right) \\
& \times\left(\Omega_{1}\right)^{-1}, \\
& \Omega_{7}=\left(-\frac{2 \epsilon^{2}\left(F_{0}-\epsilon^{2}+r_{2}^{2}\right)}{\Omega_{2}}+\frac{2 r_{2}^{2}\left(F_{0}-\epsilon^{2}+r_{2}^{2}\right)}{\Omega_{2}}\right), \\
& \Omega_{8}=\frac{2 \epsilon^{2}\left(F_{0}-\epsilon^{2}+r_{2}^{2}\right) \log \left[r_{2}\right]}{\Omega_{2}} \\
& -\frac{2 r_{2}^{2}\left(F_{0}-\epsilon^{2}+r_{2}^{2}\right) \log [\epsilon]}{\Omega_{2}}, \\
& \Omega_{9}=\frac{2\left(F_{0}-\epsilon^{2}+r_{2}^{2}\right)\left(\log [\epsilon]-\log \left[r_{2}\right]\right)}{\Omega_{2}}, \\
& \Omega_{10}=2 \mathrm{Wi} \Omega_{4} \Omega_{7}+2 \mathrm{Wi} \Omega_{6} \Omega_{7}, \\
& \Omega_{11}=4 \mathrm{Wi} \Omega_{3} \Omega_{9}-4 \mathrm{Wi} \Omega_{4} \Omega_{9} \\
& +4 \mathrm{Wi} \Omega_{5} \Omega_{9}-4 \mathrm{Wi} \Omega_{6} \Omega_{9}, \\
& \Omega_{12}=\mathrm{Wi} \Omega_{8} \Omega_{7}^{\prime}-\mathrm{Wi} \Omega_{7}^{\prime} \text {, } \\
& \Omega_{13}=-4 \mathrm{Wi} \Omega_{4} \Omega_{9}-4 \mathrm{Wi} \Omega_{6} \Omega_{9}+\mathrm{Wi} \Omega_{9} \Omega_{7}^{\prime} \\
& -2 \mathrm{Wi} \Omega_{9}^{\prime}+2 \mathrm{Wi} \Omega_{8} \Omega_{9}^{\prime},
\end{aligned}
$$




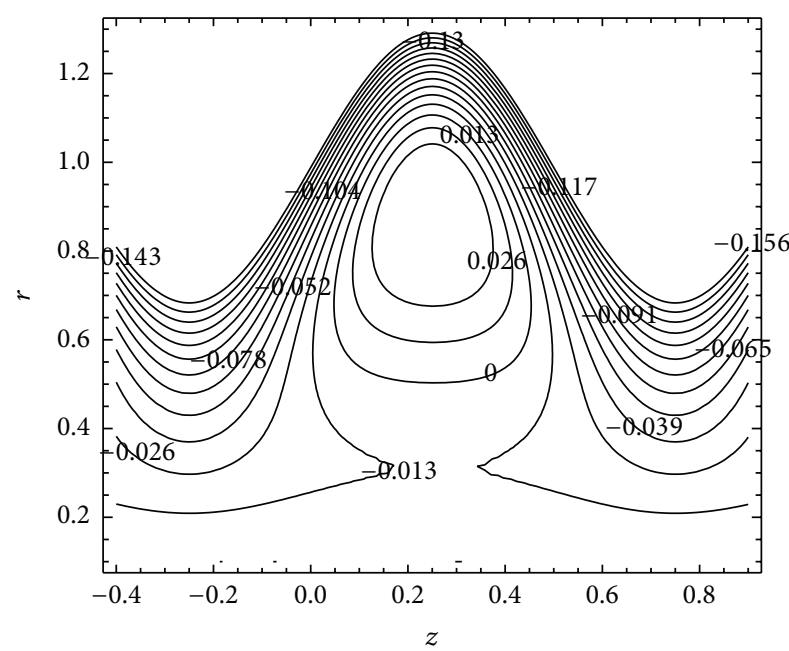

(a)

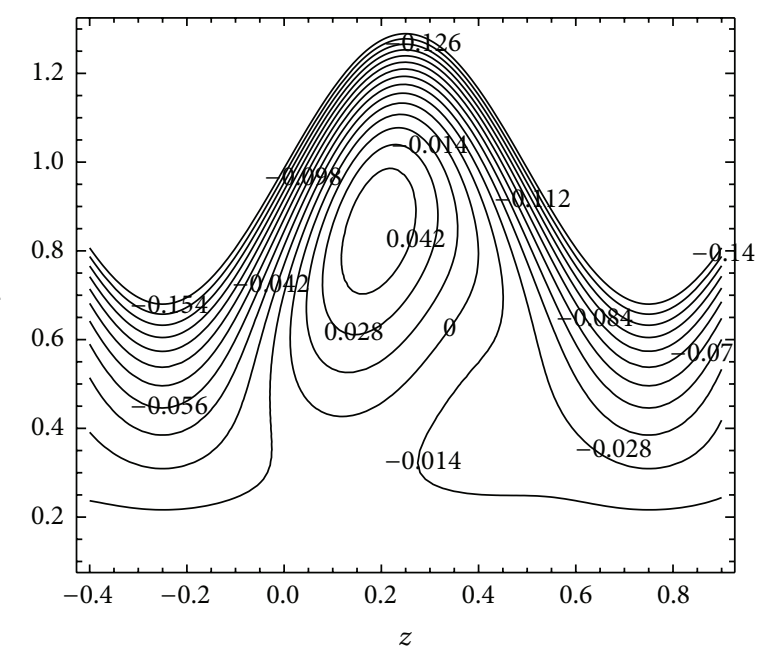

(b)

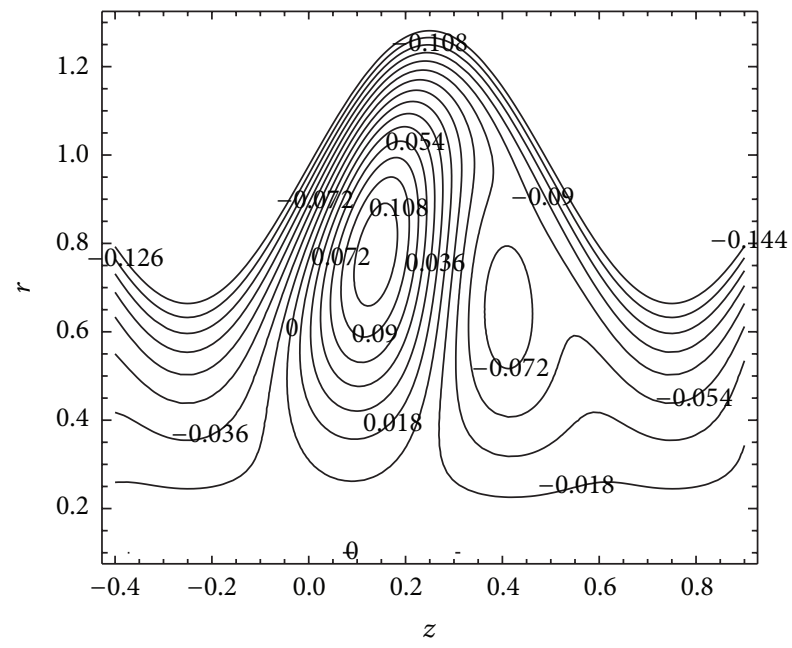

(c)

FIGURE 17: The effect of Reynolds number on the streamline patterns in the wave frame for different three values of $\operatorname{Re}:(a) \operatorname{Re}=0$, (b) $\operatorname{Re}=10$, (c) $\operatorname{Re}=50$ at $\mathrm{Wi}=0.02, \epsilon=0.1, \overline{\mathrm{Q}}=0.7, \delta=0.156$, and $\phi=0.3, r \in\left[\epsilon, r_{2}\right]$.

$$
\begin{aligned}
\Omega_{14}= & \left(2 \Omega_{5} \Omega_{9}+\Omega_{7} \Omega_{9}^{\prime}\right), \\
\Omega_{15}= & -\Omega_{10}+4 \mathrm{Wi} \Omega_{5} \Omega_{7}, \\
\Omega_{16}= & \Omega_{11}+\operatorname{Re} \Omega_{3} \Omega_{7}-4 \mathrm{Wi} \Omega_{5} \Omega_{9}, \\
\Omega_{17}= & \frac{\Omega_{13}}{2}-\frac{1}{2} \mathrm{Wi} \Omega_{14}-\frac{1}{4} \operatorname{Re} \Omega_{4} \Omega_{7} \\
& \quad-\frac{1}{4} \operatorname{Re} \Omega_{5} \Omega_{7}-\frac{1}{4} \operatorname{Re} \Omega_{6} \Omega_{7} \\
& +\frac{1}{4} \operatorname{Re} \Omega_{7}^{\prime}+\frac{3}{8} \operatorname{Re} \Omega_{7} \Omega_{7}^{\prime}-\frac{1}{4} \operatorname{Re} \Omega_{8} \Omega_{7}^{\prime} \\
& \quad-\frac{1}{4} \operatorname{Re} \Omega_{8}^{\prime}-\frac{1}{4} \operatorname{Re} \Omega_{7} \Omega_{8}^{\prime}+\frac{1}{4} \operatorname{Re} \Omega_{8} \Omega_{8}^{\prime}, \\
\Omega_{18}= & \mathrm{Wi} \Omega_{14}+\frac{1}{4} \operatorname{Re} \Omega_{5} \Omega_{7}-\frac{1}{4} \operatorname{Re} \Omega_{7}^{\prime}
\end{aligned}
$$

$$
\begin{aligned}
& -\frac{1}{2} \operatorname{Re} \Omega_{7} \Omega_{7}^{\prime}+\frac{1}{4} \operatorname{Re} \Omega_{8} \Omega_{7}^{\prime}+\frac{1}{4} \operatorname{Re} \Omega_{7} \Omega_{8}^{\prime}, \\
\Omega_{19}= & \frac{1}{8} \operatorname{Re} \Omega_{4} \Omega_{9}-\frac{1}{16} \operatorname{Re} \Omega_{5} \Omega_{9}-\frac{1}{8} \operatorname{Re} \Omega_{6} \Omega_{9} \\
& -\frac{1}{32} \operatorname{Re} \Omega_{9} \Omega_{7}^{\prime}+\frac{1}{16} \operatorname{Re} \Omega_{9} \Omega_{8}^{\prime}-\frac{1}{16} \operatorname{Re} \Omega_{9}^{\prime} \\
& -\frac{1}{32} \operatorname{Re} \Omega_{7} \Omega_{9}^{\prime}+\frac{1}{16} \operatorname{Re} \Omega_{8} \Omega_{9}^{\prime}+\frac{1}{2} \mathrm{Wi} \Omega_{9} \Omega_{9}^{\prime}, \\
\Omega_{20}= & \left.\frac{1}{8} \operatorname{Re} \Omega_{5} \Omega_{9}+\frac{1}{16} \operatorname{Re} \Omega_{9} \Omega_{7}^{\prime}+\frac{1}{16} \operatorname{Re} \Omega_{7} \Omega_{9}^{\prime}\right), \\
\Omega_{21}= & \epsilon^{2} \Omega_{17}-\frac{\epsilon^{2} \log [\epsilon] \Omega_{17}}{-\log [\epsilon]+\log \left[r_{2}\right]} \\
& +\frac{r_{2}^{2} \log [\epsilon] \Omega_{17}}{-\log [\epsilon]+\log \left[r_{2}\right]}-\epsilon^{2} \log [\epsilon] \Omega_{18}
\end{aligned}
$$




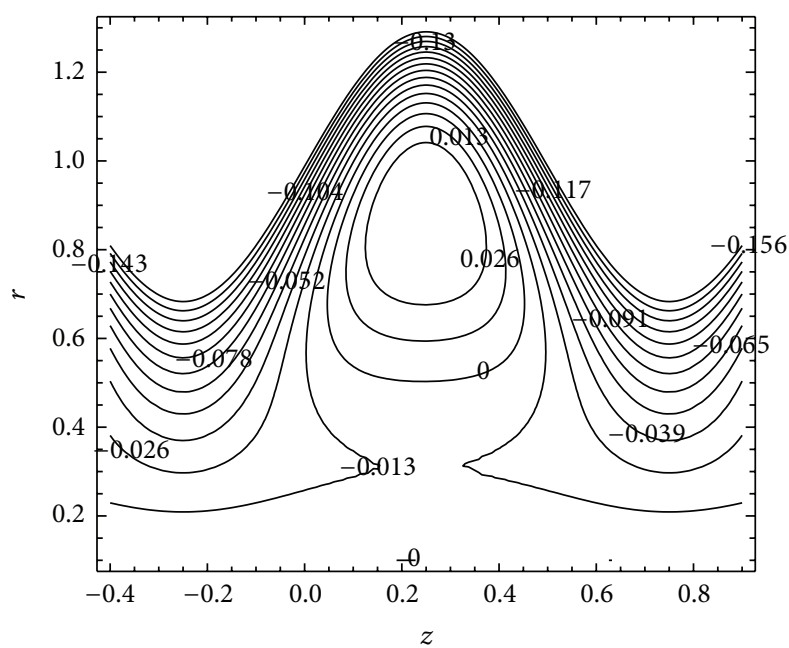

(a)

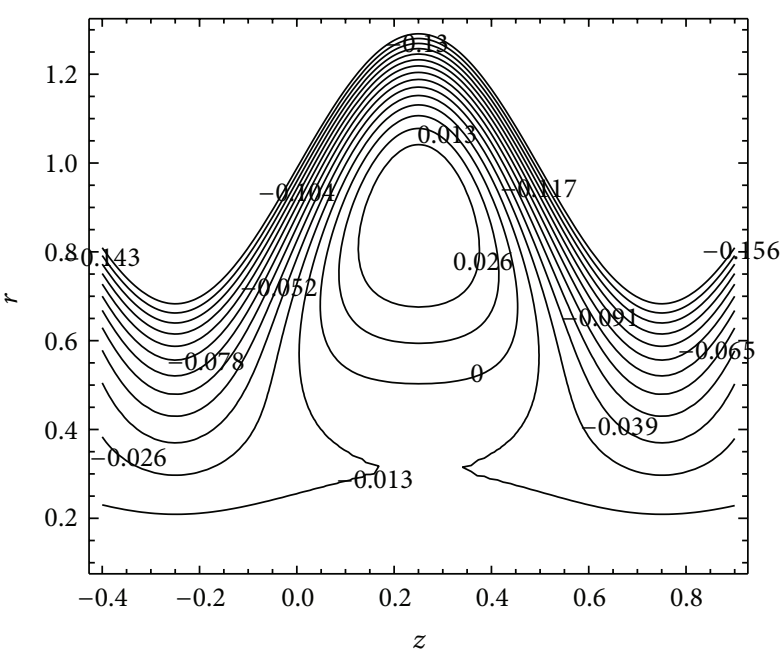

(b)

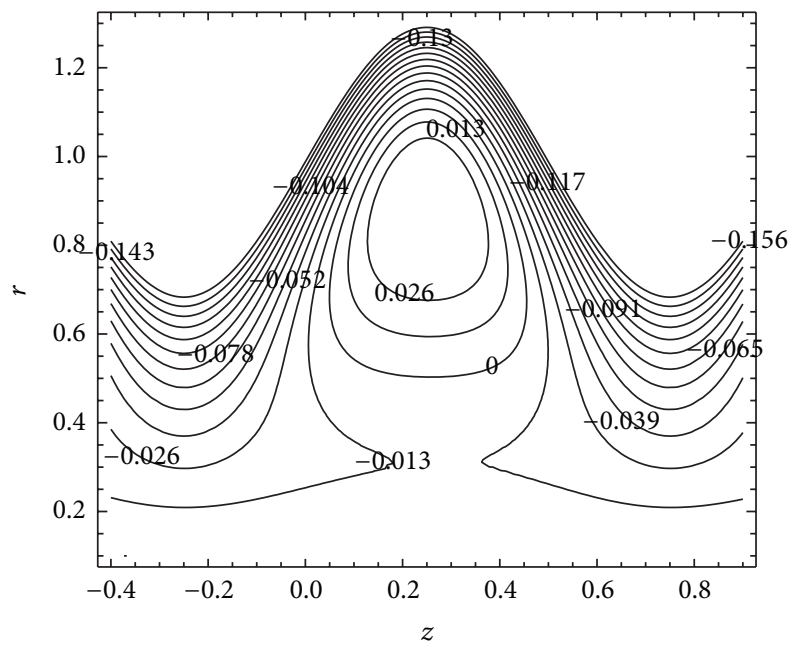

(c)

FIGURE 18: Graph of streamlines for different three values of Wi: (a) $\mathrm{Wi}=0$, (b) $\mathrm{Wi}=0.04$, (c) $\mathrm{Wi}=0.08$ at $\epsilon=0.1, \overline{\mathrm{Q}}=0.7, \delta=0.156$, $\mathrm{Re}=0.1$, and $\phi=0.3, r \in\left[\epsilon, r_{2}\right]$.

$$
\begin{aligned}
& -\frac{\epsilon^{2} \log [\epsilon]^{2} \Omega_{18}}{-\log [\epsilon]+\log \left[r_{2}\right]}+\frac{r_{2}^{2} \log [\epsilon] \log \left[r_{2}\right] \Omega_{18}}{-\log [\epsilon]+\log \left[r_{2}\right]} \\
& -\epsilon^{4}\left(\Omega_{19}+\log [\epsilon] \Omega_{20}\right) \\
& -\frac{\epsilon^{4} \log [\epsilon]\left(\Omega_{19}+\log [\epsilon] \Omega_{20}\right)}{-\log [\epsilon]+\log \left[r_{2}\right]} \\
& +\frac{r_{2}^{4} \log [\epsilon]\left(\Omega_{19}+\log \left[r_{2}\right] \Omega_{20}\right)}{-\log [\epsilon]+\log \left[r_{2}\right]} \\
& -\frac{\Omega_{15}+2 \mathrm{Wi} \log [\epsilon] \Omega_{5} \Omega_{7}}{\epsilon} \\
& -\frac{\log [\epsilon]\left(\Omega_{15}+2 \mathrm{Wi} \log [\epsilon] \Omega_{5} \Omega_{7}\right)}{\epsilon\left(-\log [\epsilon]+\log \left[r_{2}\right]\right)}
\end{aligned}
$$$$
+\frac{\log [\epsilon]\left(\Omega_{15}+2 \mathrm{Wi} \log \left[r_{2}\right] \Omega_{5} \Omega_{7}\right)}{r_{2}\left(-\log [\epsilon]+\log \left[r_{2}\right]\right)}
$$$$
-\frac{2}{9} \operatorname{Re} \epsilon^{3} \Omega_{3} \Omega_{9}-\frac{2 \operatorname{Re} \epsilon^{3} \log [\epsilon] \Omega_{3} \Omega_{9}}{9\left(-\log [\epsilon]+\log \left[r_{2}\right]\right)}
$$$$
+\frac{2 \operatorname{Re} r_{2}^{3} \log [\epsilon] \Omega_{3} \Omega_{9}}{9\left(-\log [\epsilon]+\log \left[r_{2}\right]\right)}
$$$$
-\epsilon\left(\Omega_{16}+4 \mathrm{Wi} \log [\epsilon] \Omega_{5} \Omega_{9}\right)
$$$$
-\frac{\epsilon \log [\epsilon]\left(\Omega_{16}+4 \mathrm{Wi} \log [\epsilon] \Omega_{5} \Omega_{9}\right)}{-\log [\epsilon]+\log \left[r_{2}\right]}
$$$$
+\frac{r_{2} \log [\epsilon]\left(\Omega_{16}+4 \mathrm{Wi} \log \left[r_{2}\right] \Omega_{5} \Omega_{9}\right)}{-\log [\epsilon]+\log \left[r_{2}\right]}
$$ 


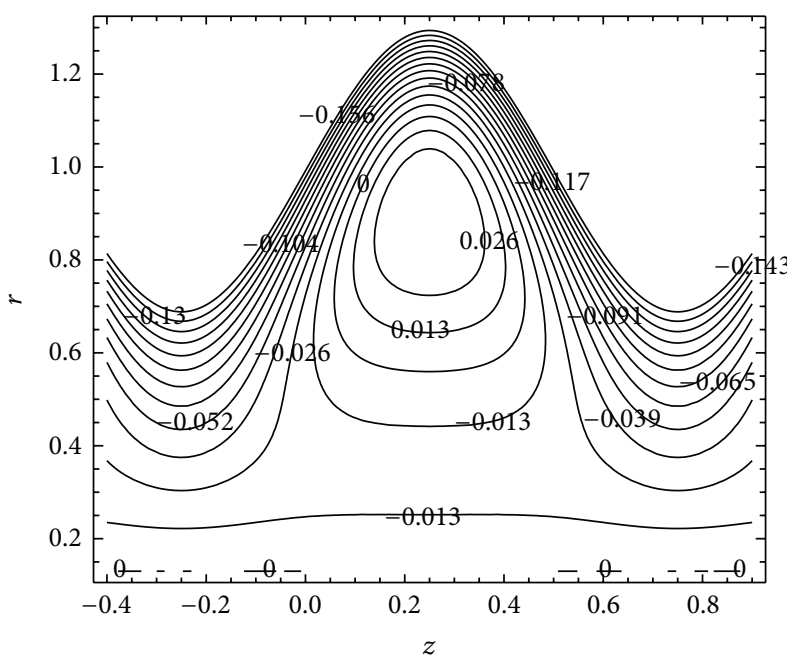

(a)

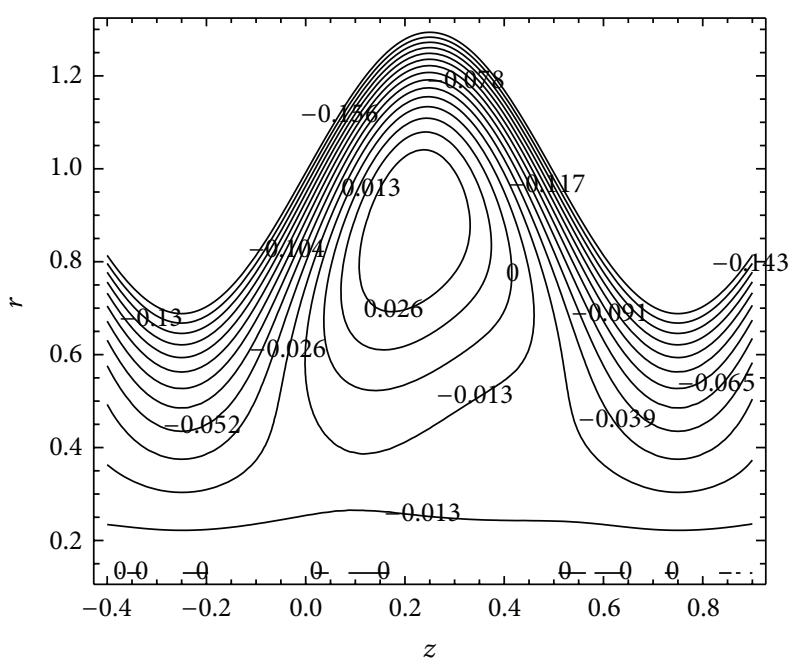

(b)

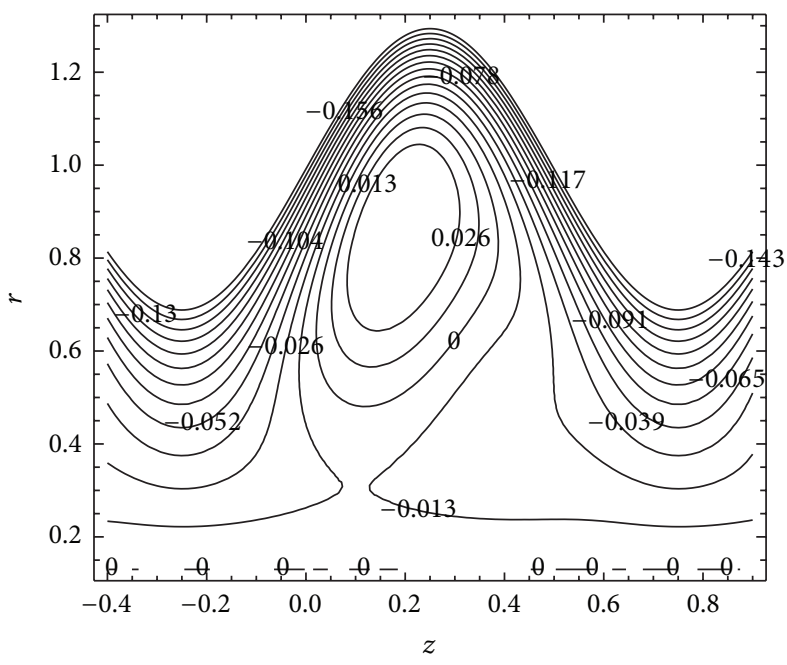

(c)

FIGURE 19: Graph of streamlines for different three values of $\delta$ : (a) $\delta=0$, (b) $\delta=0.08$, (c) $\delta=0.156$ at $\epsilon=0.13, \bar{Q}=0.7, \mathrm{Wi}=0.05, \operatorname{Re}=10$, and $\phi=0.3, r \in\left[\epsilon, r_{2}\right]$.

$$
\begin{aligned}
& -\frac{1}{2} \mathrm{Wi} \log [\epsilon]^{2} \Omega_{7} \Omega_{7}^{\prime} \\
& -\frac{1}{4} \operatorname{Re} \epsilon^{2} \log [\epsilon]^{2} \Omega_{7} \Omega_{7}^{\prime} \\
& -\frac{\mathrm{Wi} \log [\epsilon]^{3} \Omega_{7} \Omega_{7}^{\prime}}{2\left(-\log [\epsilon]+\log \left[r_{2}\right]\right)} \\
& -\frac{\operatorname{Re} \epsilon^{2} \log [\epsilon]^{3} \Omega_{7} \Omega_{7}^{\prime}}{4\left(-\log [\epsilon]+\log \left[r_{2}\right]\right)} \\
& +\frac{\mathrm{Wi} \log [\epsilon] \log \left[r_{2}\right]^{2} \Omega_{7} \Omega_{7}^{\prime}}{2\left(-\log [\epsilon]+\log \left[r_{2}\right]\right)} \\
& +\frac{\operatorname{Re} r_{2}^{2} \log [\epsilon] \log \left[r_{2}\right]^{2} \Omega_{7} \Omega_{7}^{\prime}}{4\left(-\log [\epsilon]+\log \left[r_{2}\right]\right)}-\frac{1}{36} \operatorname{Re} \epsilon^{6} \Omega_{9} \Omega_{9}^{\prime}
\end{aligned}
$$




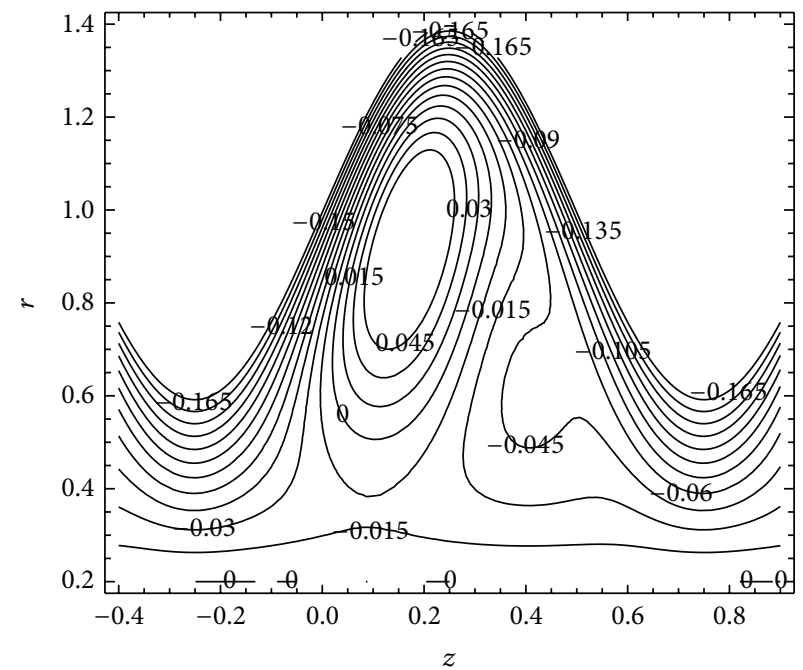

(a)

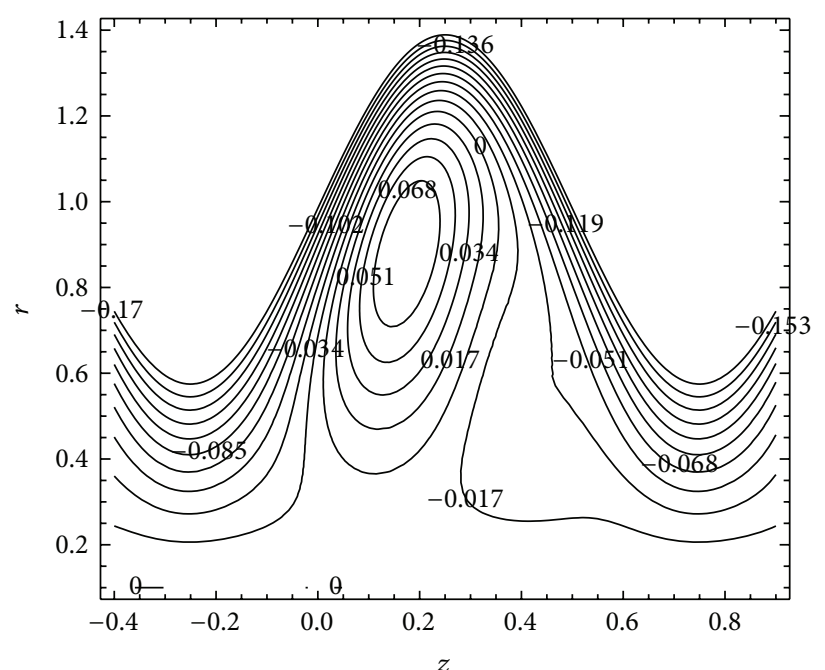

(b)

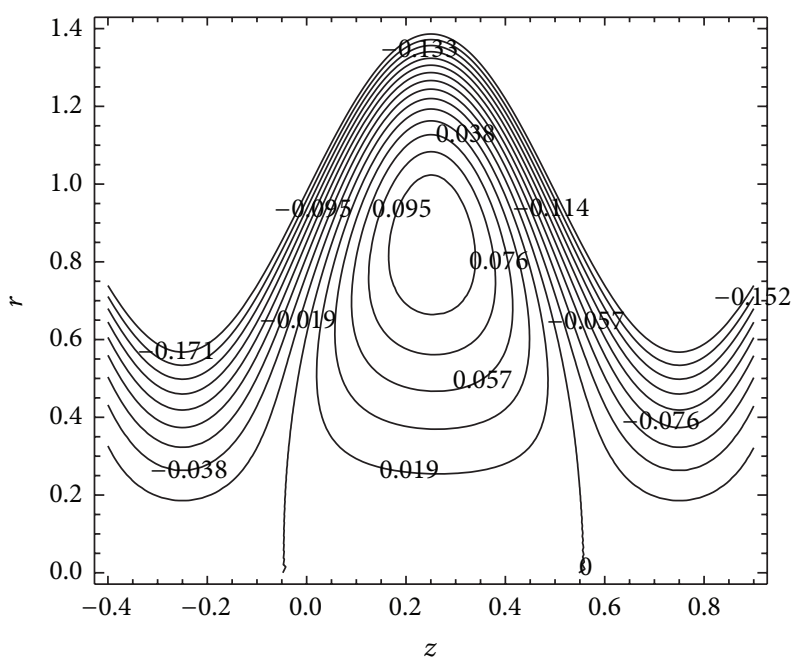

(c)

FIGURE 20: Graph of streamlines for different three values of $\epsilon$ : (a) $\epsilon=0.2$, (b) $\epsilon=0.1$, (c) $\epsilon=0.0$ at $\delta=0.156, \bar{Q}=0.7, \mathrm{Wi}=0.05, \operatorname{Re}=15$, and $\phi=0.4, r \in\left[\epsilon, r_{2}\right]$.

$$
\begin{aligned}
& +\frac{\Omega_{15}+2 \mathrm{Wi} \log \left[r_{2}\right] \Omega_{5} \Omega_{7}}{r_{2}} \\
& -\frac{2}{9} \operatorname{Re} \epsilon^{3} \Omega_{3} \Omega_{9}+\frac{2}{9} \operatorname{Re} r_{2}^{3} \Omega_{3} \Omega_{9} \\
& -\epsilon\left(\Omega_{16}+4 \mathrm{Wi} \log [\epsilon] \Omega_{5} \Omega_{9}\right) \\
& +r_{2}\left(\Omega_{16}+4 \mathrm{Wi} \log \left[r_{2}\right] \Omega_{5} \Omega_{9}\right) \\
& -\frac{1}{2} \mathrm{Wi} \log [\epsilon]^{2} \Omega_{7} \Omega_{7}^{\prime} \\
& -\frac{1}{4} \operatorname{Re} \epsilon^{2} \log [\epsilon]^{2} \Omega_{7} \Omega_{7}^{\prime} \\
& +\frac{1}{2} \mathrm{Wi} \log \left[r_{2}\right]^{2} \Omega_{7} \Omega_{7}^{\prime}
\end{aligned}
$$

$$
\begin{aligned}
& +\frac{1}{4} \operatorname{Re} r_{2}^{2} \log \left[r_{2}\right]^{2} \Omega_{7} \Omega_{7}^{\prime}-\frac{1}{36} \operatorname{Re} \epsilon^{6} \Omega_{9} \Omega_{9}^{\prime} \\
& \left.+\frac{1}{36} \operatorname{Re} r_{2}^{6} \Omega_{9} \Omega_{9}^{\prime}\right), \\
c_{6}=-\frac{1}{\epsilon}[- & \frac{1}{36} \epsilon^{6}\left(6 \Omega_{19}^{\prime}-\Omega_{20}^{\prime}\right) \\
& -\epsilon\left(\Omega_{15}^{\prime}-2 \mathrm{Wi}\left(\Omega_{7} \Omega_{5}^{\prime}+\Omega_{5} \Omega_{7}^{\prime}\right)\right) \\
& -\frac{2}{45} \operatorname{Re} \epsilon^{5}\left(\Omega_{9} \Omega_{3}^{\prime}+\Omega_{3} \Omega_{9}^{\prime}\right) \\
& -\frac{1}{9} \epsilon^{3}\left(3 \Omega_{16}^{\prime}-4 \mathrm{Wi}\left(\Omega_{9} \Omega_{5}^{\prime}+\Omega_{5} \Omega_{9}^{\prime}\right)\right)
\end{aligned}
$$




$$
\begin{aligned}
& -\frac{1}{16} \epsilon^{2}\left(4 \mathrm{Wi}+\operatorname{Re} \epsilon^{2}\right) \log [\epsilon]^{2}\left(\Omega_{7}^{\prime 2}+\Omega_{7} \Omega_{7}^{\prime \prime}\right) \\
& -\frac{1}{128} \epsilon^{4}\left(32 \Omega_{17}^{\prime}-8 \Omega_{18}^{\prime}+\operatorname{Re}\left(\Omega_{7}^{\prime 2}+\Omega_{7} \Omega_{7}^{\prime \prime}\right)\right) \\
& -\frac{\epsilon^{2}}{\left(16 r_{2}\left(\log \left(\epsilon / r_{2}\right)\right)^{2}\right)} \\
& \times\left(\frac{d p_{1}}{d z}(1+2 \log [\epsilon])\right. \\
& \times\left(\epsilon^{2}+r_{2}^{2}\left(-1-2 \log \frac{\epsilon}{r_{2}}\right)\right) r_{2}^{\prime} \\
& +2 r_{2}\left(\log \frac{\epsilon}{r_{2}}\right)^{2} \\
& \left.\times\left(4 \Omega_{21}^{\prime}-2 \Omega_{22}^{\prime}+\mathrm{Wi}\left(\Omega_{7}^{\prime 2}+\Omega_{7} \Omega_{7}^{\prime \prime}\right)\right)\right) \\
& -\frac{\log [\epsilon]}{\left(8 r_{2}\left(\log \left(\epsilon / r_{2}\right)\right)^{2}\right)} \\
& \times\left(\frac{1}{6} \epsilon^{6} \Omega_{20}^{\prime}+2 \mathrm{Wi} \epsilon\left(\Omega_{7} \Omega_{5}^{\prime}+\Omega_{5} \Omega_{7}^{\prime}\right)\right. \\
& +\frac{4}{3} \mathrm{Wi} \epsilon^{3}\left(\Omega_{9} \Omega_{5}^{\prime}+\Omega_{5} \Omega_{9}^{\prime}\right) \\
& +\frac{\epsilon^{4}}{32} \epsilon^{4}\left(8 \Omega_{18}^{\prime}-\operatorname{Re}\left(\Omega_{7}^{\prime 2}+\Omega_{7} \Omega_{7}^{\prime \prime}\right)\right) \\
& +\left(\epsilon ^ { 2 } \left(\frac{d p_{1}}{d z}\left(-\epsilon^{2}+r_{2}^{2}\left(1+2 \log \frac{\epsilon}{r_{2}}\right)\right) r_{2}^{\prime}\right.\right. \\
& +2 r_{2}\left(\log \frac{\epsilon}{r_{2}}\right)^{2} \\
& \times\left(2 \Omega_{22}^{\prime}\right. \\
& \left.\left.\left.-\mathrm{Wi}\left(\Omega_{7}^{\prime 2}+\Omega_{7} \Omega_{7}^{\prime \prime}\right)\right)\right)\right) \\
& \left.-\frac{1}{288} \operatorname{Re} \epsilon^{8}\left(\Omega_{9}^{\prime 2}+\Omega_{9} \Omega_{9}^{\prime \prime}\right)\right] .
\end{aligned}
$$

\section{Conflict of Interests}

The authors declare that they have no conflict of interests regarding the publication of this paper.

\section{Acknowledgments}

The authors are grateful to the referees and editors for their constructive suggestions. This paper was funded by the Deanship of Scientific Research (DSR), King Abdulaziz University, Jeddah, under Grant no. 66-130-D1432. The authors, therefore, acknowledge with thanks DSR technical and financial support.

\section{References}

[1] T. W. Latham, Fluid motion in a peristaltic pump [M.Sc. thesis], Massachusetts Institute of Technology, Cambridge, Mass, USA, 1966.

[2] A. H. Shapiro, M. Y. Jaffrin, and S. L. Weinberg, "Peristaltic pumping with long wavelength at low Reynolds number," Journal of Fluid Mechanics, vol. 37, no. 4, pp. 799-825, 1969.

[3] T. F. Zien and S. A. Ostrach, "A long wave approximation to peristaltic motion," Journal of Biomechanics, vol. 3, no. 1, pp. 6375, 1970.

[4] R. A. Ramachandra and S. Usha, "Peristaltic transport of two immiscible viscous fluids in a circular tube," Journal of Fluid Mechanics, vol. 298, pp. 271-285, 1995.

[5] Kh. S. Mekheimer, E. F. El Shehawey, and A. M. Elaw, "Peristaltic motion of a particle-fluid suspension in a planar channel," International Journal of Theoretical Physics, vol. 37, no. 11, pp. 2895-2920, 1998.

[6] R. B. Bird, R. G. Armstrong, and O. Hassager, Dynamics of Polymeric Liquids, Fluid Mechanics, vol. 1, John Wiley \& Sons, New York, NY, USA, 1987.

[7] J. G. Oldroyd, "On the formulation of rheological equations of state," Proceedings of the Royal Society of London A: Mathematical, Physical and Engineering Sciences, vol. 200, pp. 523-541, 1950.

[8] T. Hayat, A. Afsar, M. Khan, and S. Asghar, "Peristaltic transport of a third order fluid under the effect of a magnetic field," Computers \& Mathematics with Applications, vol. 53, no. 7, pp. 1074-1087, 2007.

[9] Y. Abd elmaboud and Kh. S. Mekheimer, "Non-linear peristaltic transport of a second-order fluid through a porous medium," Applied Mathematical Modelling, vol. 35, no. 6, pp. 2695-2710, 2011.

[10] A. E. H. Abd El Naby, A. E. M. El Misery, and M. F. Abd El Kareem, "Effects of a magnetic field on trapping through peristaltic motion for generalized Newtonian fluid in channel," Physica A: Statistical Mechanics and Its Applications, vol. 367, pp. 79-92, 2006.

[11] T. Hayat, M. Khan, A. M. Siddiqui, and S. Asghar, "Nonlinear peristaltic flow of a non-Newtonian fluid under effect of a magnetic field in a planar channel," Communications in Nonlinear Science and Numerical Simulation, vol. 12, no. 6, pp. 910-919, 2007.

[12] D. Tsiklauri and I. Beresnev, "Non-Newtonian effects in the peristaltic flow of a Maxwell fluid," Physical Review E, vol. 64, no. 2, part 2, Article ID 036303, 2001.

[13] Kh. S. Mekheimer and A. N. Abdel-Wahab, "Net annulus flow of a compressible viscous liquid with peristalsis," Journal of Aerospace Engineering, vol. 25, no. 4, pp. 660-669, 2012.

[14] A. M. Siddiqui and W. H. Schwarz, "Peristaltic flow of a second-order fluid in tubes," Journal of Non-Newtonian Fluid Mechanics, vol. 53, pp. 257-284, 1994.

[15] Kh. S. Mekheimer and A. N. Abdel-Wahab, "Compressibility effects on peristaltic flow of a non-Newtonian Maxwell fluid through an annulus," in Fluid Transport: Theory, Dynamics and Transport, pp. 219-235, Nova Science Publishers, 2011. 
[16] K. Vajravelu, S. Sreenadh, and V. R. Babu, "Peristaltic transport of a Herschel-Bulkley fluid in an inclined tube," International Journal of Non-Linear Mechanics, vol. 40, no. 1, pp. 83-90, 2005.

[17] A. V. Mernone and J. N. Mazumdar, "A mathematical study of peristaltic transport of a casson fluid," Mathematical and Computer Modelling, vol. 35, no. 7-8, pp. 895-912, 2002.

[18] Kh. S. Mekheimer, "Peristaltic transport of a Newtonian fluid through a uniform and non-uniform annulus," The Arabian Journal for Science and Engineering, vol. 30, no. 1A, p. 69, 2005.

[19] A. E. H. Abd El Naby and A. E. M. El Misiery, "Effects of an endoscope and generalized Newtonian fluid on peristaltic motion," Applied Mathematics and Computation, vol. 128, no. 1, pp. 19-35, 2002.

[20] A. E. H. Abd El Naby, A. E. M. El Misery, and I. I. El Shamy, "Effects of an endoscope and fluid with variable viscosity on peristaltic motion," Applied Mathematics and Computation, vol. 158, no. 2, pp. 497-511, 2004.

[21] T. Hayat, N. Ali, S. Asghar, and A. M. Siddiqui, "Exact peristaltic flow in tubes with an endoscope," Applied Mathematics and Computation, vol. 182, no. 1, pp. 359-368, 2006.

[22] Kh. S. Mekheimer and Y. Abd Elmaboud, "Peristaltic flow through a porous medium in an annulus: application of an endoscope," Applied Mathematics \& Information Sciences, vol. 2, no. 1, pp. 103-121, 2008.

[23] A. Chorin and J. Marsden, A Mathematical Introduction to Fluid Mechanics, Springer, New York, NY, USA, 1997.

[24] R. L. Burden and J. D. Faires, Numerical Analysis, Brooks Cole, Florence, Ky, USA, 9th edition, 2010. 


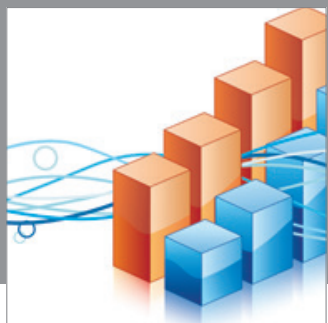

Advances in

Operations Research

mansans

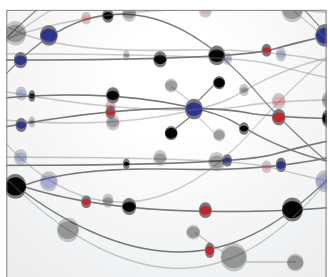

The Scientific World Journal
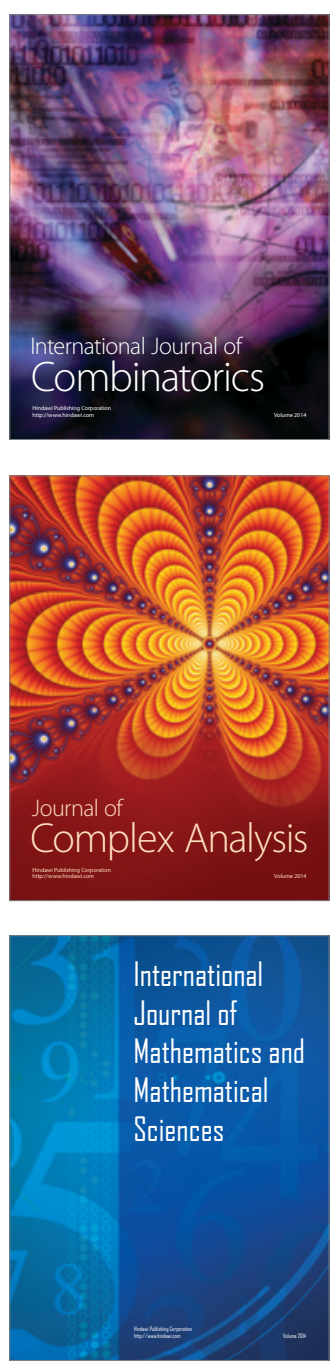
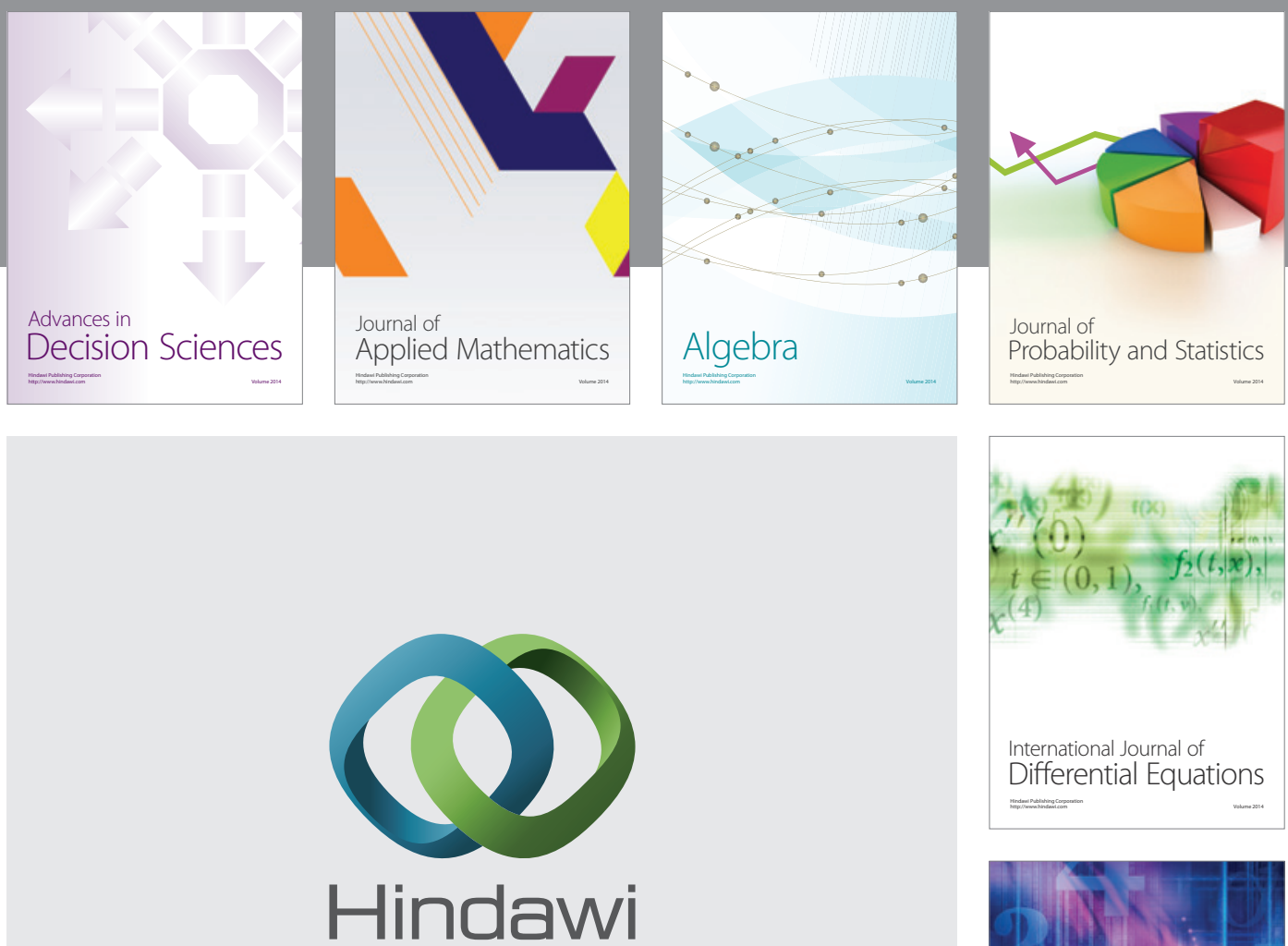

Submit your manuscripts at http://www.hindawi.com
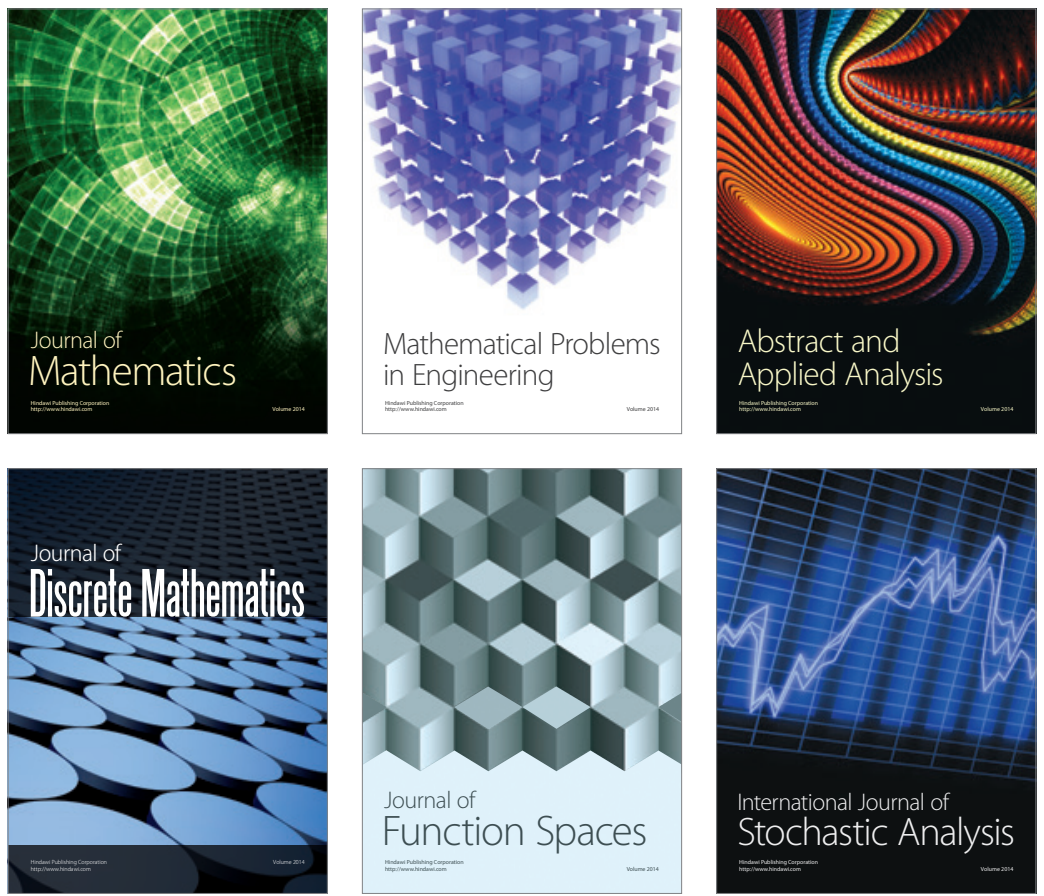

Journal of

Function Spaces

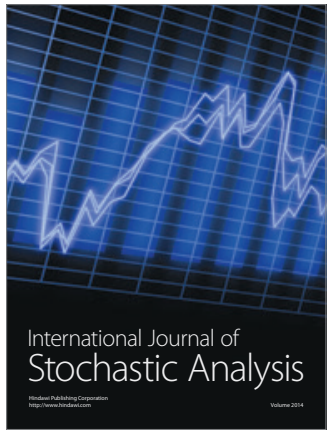

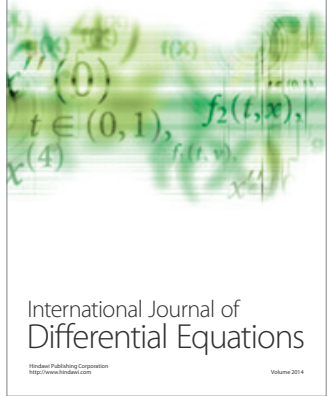
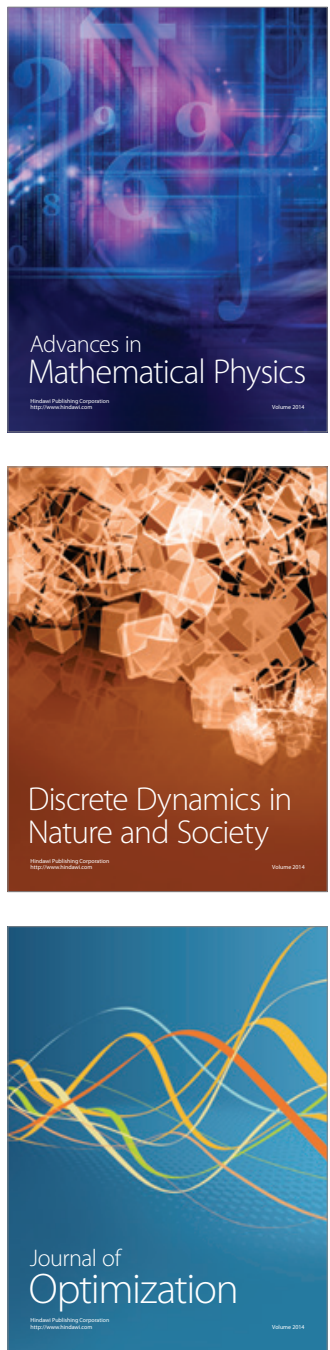